\title{
Spectroscopic diagnostics of stellar transition regions and coronae in the XUV: AU Mic in quiescence
}

\author{
G. Del Zanna ${ }^{1}$, M. Landini ${ }^{2}$, and H. E. Mason ${ }^{1}$ \\ 1 Department of Applied Mathematics and Theoretical Physics, University of Cambridge, UK \\ 2 Dipartimento di Astronomia e Scienza dello Spazio, Università di Firenze, Italy
}

Received 17 October 2001 / Accepted 29 January 2002

\begin{abstract}
In this paper we review the spectroscopic diagnostic techniques that can be applied to XUV observations of active stars. In particular, we discuss methods for the determination of electron densities, emission measures and the chemical composition of the stellar transition regions and coronae. We pay particular attention to the atomic data used and we revise several previous analyses with the most recent atomic data (from CHIANTI). We find severe limitations to some of the commonly used methods and atomic data and we obtain results which are significantly different from other authors. We illustrate this with a number of examples. We analyse a combined set of multi-wavelength observations (EUVE, HST/STIS, FUSE) for the quiescent phase of the dMe star AU Mic. A major conclusion of this paper is that the use of spectral lines from the $\mathrm{Li}$ and $\mathrm{Na}$ isoelectronic sequences, common in previous literature, produces erroneous results in the determination of emission measures, elemental abundances and transition region densities.
\end{abstract}

Key words. techniques: spectroscopic - atomic data - stars: atmospheres - stars: individual: AU Mic

\section{Introduction}

Many of the bright sources in the XUV are late-type dwarf active stars with very strong chromospheric emission. These stars have quiescent XUV/bolometric luminosity ratios many orders of magnitude greater than the Sun. They also present recurrent flares. The physical characteristics of the stellar transition regions (TRs) and coronae provide important observational constraints on the modeling of the thermal structure of their outer atmospheres (see, e.g., Jordan 2000). It is important to determine: the thermal structure of the stellar outer atmospheres, in terms of the differential emission measure $D E M$ or emission measure $E M$; the chemical composition of the TR and corona; electron densities (or pressures); radiative losses. At present, these physical characteristics are poorly known for active stars. It is important to measure them, because they seem to vary from star to star, and to be different from the Sun. For example, EUV observations indicate that some stars have coronal abundances similar to the Sun, while other stars do not (see, e.g., the review of Feldman \& Laming 2000). This could have important implications for our understanding of the various processes taking place in stellar atmospheres.

Send offprint requests to: G. Del Zanna,

e-mail: G.Del-Zanna@damtp.cam.ac.uk
Spectroscopic diagnostics can be applied to XUV observations to determine the above physical characteristics. Until recently, low- to medium-resolution XUV spectroscopy provided measurements which were subject to large uncertainties, with approximate methods being used to analyse them. For example: electron densities were estimated from emission measures; the emission measures were estimated assuming an isothermal or a twotemperature coronal plasma; elemental abundances were estimated using global fits to the spectra. The advent of high-resolution XUV spectroscopy in the past few years (summarised in Sect. 2) provides an excellent opportunity to determine these physical parameters more directly and more accurately.

A large body of literature has been written on the subject of spectroscopic diagnostics (for a general review see, e.g., Mason \& Monsignori Fossi 1994) and applications to solar and stellar observations. Unfortunately, in many studies, particularly those based on stellar observations, incorrect physical parameters have been obtained. This has in turn led to unnecessary physical conjectures that we believe should now be revisited. In this paper, we are primarily concerned with pointing out the reasons why wrong results have been obtained. We illustrate this with some examples and suggest the correct methodologies that should be applied to high-resolution XUV stellar spectra. In many cases, inaccurate atomic calculations have been 
used. The problematics discussed here are more generally applicable, both for solar and stellar atmospheres.

It is important to realise that some of the physical parameters are inter-related. For example, an accurate knowledge of the $D E M$ is necessary to estimate elemental abundances (see Sect. 3), and both the DEM and the chemical composition are important factors in the calculation of the radiative losses (cf. Cook et al. 1989). The $D E M$ and the relative element abundances can only be directly obtained to a high accuracy from the line intensities if the spectral lines:

- (a) are obtained from high signal to noise $(S / N)$ observations with a high resolving power;

- (b) are optically thin;

- (c) are density-insensitive;

- (d) cover a large temperature range, from transition region up to coronal and flare temperatures;

- (e) cover a large range of elements and ions;

- (f) are from various isoelectronic sequences;

- (g) have corresponding accurate atomic data.

Point (a) is self evident. Point (b) does not mean that lines that are not optically thin cannot be used for diagnostics. If the optical depths can be estimated (see Sect. 5.1) and are not large, then the lines can still be used without the need for full radiative transfer calculations. Point (c) is often overlooked. Point (d) is particularly important for an accurate calculation of the radiative losses. A major problem with past and present spectroscopic data from various satellites is that they observe spectral lines that are emitted over a restricted temperature range, and do not usually satisfy all the above requirements (see Sect. 2). Point (e) is important for the derivation of elemental abundances.

Regarding point (f), anomalous behaviour of lines emitted by some ions from the $\mathrm{Li}$ and $\mathrm{Na}$ isoelectronic sequences has now been reported by several authors (see Sect. 3.1). However, in most of the literature (see, e.g., Brown et al. 1984b; Brown et al. 1984a; Hartmann et al. 1985; Jordan et al. 1985; Byrne et al. 1987; Jordan et al. 1987; Linsky et al. 1989; Quin et al. 1993; Maran et al. 1994; Linsky et al. 1995; Doschek 1997; Griffiths \& Jordan 1998; Brandt et al. 2001), these lines have been used for solar and stellar emission measure analyses, since they are among the most prominent ones in the FUV. In this paper we show that erroneous results are obtained if these lines are used, in particular in terms of $D E M$, elemental abundances and electron densities. For example, many authors have used the EM loci method (see Sect. 3.3 for details) and lines with anomalous behaviour to determine electron densities. The values derived from the $E M$ loci method are often one order of magnitude higher than those derived with the line ratio technique. In the majority of cases the authors adopt the results obtained from the $E M$ loci method, because of the weakness of the lines used in the density-sensitive line ratios, and the various uncertainties due to blending and atomic data. The discrepancy in the electron densities derived from the two different methods is often explained with unnecessary conjectures, for example by assuming that the emission was formed in distinct types of atmospheric structures, having different densities (see, e.g., Linsky et al. 1995; Pagano et al. 2000). In this paper we show that the lower densities found by the line ratio technique should be adopted and that the two methods are fully consistent if the correct $E M$ is obtained.

In Sect. 2 we briefly discuss some of the limitations and diagnostic possibilities offered by observations with previous and current XUV spectroscopic instruments, from the the TR through to the corona. Section 3 contains a discussion of diagnostics in the far ultraviolet spectral region (FUV: $900-1700 \AA$ ) for the determination of the physical parameters of the transition regions in active stars. In Sect. 4 we consider multi-wavelength observations of the quiescent phase of the dMe star AU Mic as a benchmark case. Section 5 presents the results, including a detailed discussion of densities, and a revision of some previous solar and stellar measurements. Section 6 draws the conclusions.

\section{Diagnostic possibilities in the XUV}

Until the launch of the recent XUV satellites, the spectral information available from stars was limited and only provided a few diagnostic possibilities. Some diagnostics of stellar coronae were possible with the Extreme Ultraviolet Explorer (EUVE) spectrometers (SW: 70-190 Aं; MW:

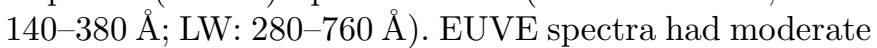
resolutions $(\Delta \lambda \approx 0.5,1$ and $2 \AA$ ) but could be used to get estimates of coronal densities from line ratios, and to constrain the $D E M$ at temperatures above a million degrees. Only a few weak lines were available at lower temperatures and for element abundance analyses (cf. Drake et al. 1995). The XMM-Newton and Chandra gratings are now providing a wealth of high-resolution X-ray spectra in the 3-170 A range. Direct estimates of temperatures, densities and element abundances are now possible using the $\mathrm{X}$-ray spectra, although all the spectroscopic diagnostics are limited to the high-temperature coronal plasma. In fact, only coronal lines emitted at temperatures above a million degrees are observed, with the exception of a few weak transition region $\mathrm{C} \mathrm{V}$ lines (formed at $\log T=5.5$ ) observed by one of the Chandra gratings.

Information on the TR can be obtained from observations in the FUV spectral region. The IUE mission observed in the FUV with two channels (SWP: 1175$2000 \AA$; LWR: 2000-3200 A). Unfortunately, IUE spectra had severely limited diagnostic capabilities, since the available lines do not simultaneously satisfy conditions (a), (b), (d), (e). IUE only observed lines formed in the chromosphere and lower TR, many of which are emitted by neutrals or singly-ionised atoms and are not opticallythin. The exceptions are a few lines of Si IV, C IV, and $\mathrm{N}$ V which unfortunately all belong to the class of anomalous behaviour. Another limiting factor was the fact that 
most lines were heavily blended, since the majority of observations were done with the SWP channel at low resolution $(\simeq 6 \AA)$.

The Goddard High Resolution Spectrograph (GHRS) and Space Telescope Imaging Spectrograph (STIS) instruments on board the NASA/ESA Hubble Space Telescope (HST) have produced very good spectroscopic data in the 117-1700 $\AA$ range, with much higher $S / N$ and spectral resolution compared to IUE. This has allowed a large number of diagnostics (in particular density) to be applied to stellar observations. However, the GHRS and STIS instruments cover similar spectral ranges to IUE, and therefore their data share some of the limitations as the IUE spectra. In fact, the lines in the GHRS and STIS spectra do not satisfy conditions (b), (c), (d), (e), (f), as discussed below. Only lines that are formed at temperatures up to $\log T=5.4$ are observed, with the exception of the Fe XXI $1354 \AA$ coronal line $(\log T=7)$. Strictly, the HST spectra on their own cannot directly provide accurate $D E M$ and elemental abundance diagnostics. Even dropping conditions (d) and (f) leaves us with just a few lines.

The Far Ultraviolet Spectroscopic Explorer (FUSE), launched in 1999, covers the $905-1187 \AA$ region with a high resolving power (20000-25000). For cool stars, the FUSE spectral range is more useful than the HST/STIS one, since it is rich in lines from different isoelectronic sequences which cover a large temperature range, from chromospheric to coronal temperatures (Fe XVIII, Fe XIX). FUSE is also the only instrument which observes the O VI doublet, important for bridging the gap between transition region and coronal temperatures. Note that also other instruments, such as the Hopkins Ultraviolet Telescope (HUT) and the Orbiting Retrievable Far and Extreme Ultraviolet Spectrometers (ORFEUS), flown on the Space Shuttle, have provided a few spectra in the FUSE spectral region, although with much lower resolution.

\section{Spectroscopic techniques for the determination of densities, temperatures and elemental abundances}

The flux $I\left(\lambda_{i j}\right)\left(\operatorname{ergs~} \mathrm{cm}^{-2} \mathrm{~s}^{-1}\right)$, of an optically thin spectral line of wavelength $\lambda_{i j}$ is

$I\left(\lambda_{i j}\right)=\frac{h \nu_{i j}}{4 \pi d^{2}} \int_{V} N_{j}\left(X^{+r}\right) A_{j i} \mathrm{~d} V$

where: $i, j$ are the lower and upper levels; $A_{j i}$ is the spontaneous transition probability; $N_{j}\left(X^{+r}\right)$ is the number density of the upper level $j$ of the emitting ion $X^{+r} ; d$ is the star's distance; $\mathrm{d} V$ is the volume element, and $V$ is the entire source volume. The flux can be written:

$I\left(\lambda_{i j}\right)=\frac{1}{d^{2}} \int_{V} G\left(N_{\mathrm{e}}, T, \lambda_{i j}\right) N_{\mathrm{e}} N_{\mathrm{H}} \mathrm{d} V$

having defined $N_{\mathrm{e}}$ and $N_{\mathrm{H}}\left(\mathrm{cm}^{-3}\right)$ as the electron and hydrogen number densities, and the contribution function $G\left(T, \lambda_{i j}, N_{\mathrm{e}}\right)\left(\operatorname{ergs~} \mathrm{cm}^{3} \mathrm{~s}^{-1}\right)$ of each line:

$G\left(N_{\mathrm{e}}, T, \lambda_{i j}\right)=A_{j i} \frac{h \nu_{i j}}{4 \pi} A_{\mathrm{b}}(X) \frac{N_{j}\left(X^{+r}\right)}{N_{\mathrm{e}} N\left(X^{+r}\right)} \frac{N\left(X^{+r}\right)}{N(X)}$ where: $A_{\mathrm{b}}(X)=N(X) / N_{\mathrm{H}}$ is the element abundance relative to hydrogen; $N_{j}\left(X^{+r}\right) / N\left(X^{+r}\right)$ is the population of level $j$ relative to the total $N\left(X^{+r}\right)$ number density of the ion $X^{+r}$ and is a function of the electron temperature and density; $N\left(X^{+r}\right) / N(X)$ is the ionisation ratio, and is predominantly a peaked function of the temperature, confining the emission to a limited temperature range. It is common to assume that the abundance of the element $A_{\mathrm{b}}(X)$ is constant over the source volume, and to define a differential emission measure $\operatorname{DEM}(T)=N_{\mathrm{e}} N_{\mathrm{H}} \frac{\mathrm{d} h}{\mathrm{~d} T}\left(\mathrm{~cm}^{-5} \mathrm{~K}^{-1}\right)$ in order to write:

$I\left(\lambda_{i j}\right)=A_{\mathrm{b}}(X) \int C\left(T, \lambda_{i j}, N_{\mathrm{e}}\right) D E M(T) \mathrm{d} T$

where we have defined $G\left(T, N_{\mathrm{e}}\right)=A_{\mathrm{b}}(X) \times C\left(T, N_{\mathrm{e}}\right)$, and assumed filling factors of unity and spherical symmetry, i.e. $\mathrm{d} V=4 \pi R_{*}^{2} \mathrm{~d} h$ ( $h$ is the coordinate along the line of sight and $R_{*}$ is the star's radius). Given a set of observed fluxes, the problem is to invert a set of integral equations like Eq. (4). In this paper, the $D E M$ analysis was performed using a modified version of the Arcetri inversion code (Monsignori Fossi \& Landini 1991). The CHIANTI atomic database (Dere et al. 1997) has been used to calculate the contribution functions of the observed lines, assuming collisional ionisation equilibrium. In particular, Version 3 of the database (Dere et al. 2001) was used, together with an update for $\mathrm{O} \mathrm{V}$ that is described below.

Note that the determination of the $\operatorname{DEM}(T)$ distribution is an ill-posed problem (see, e.g. Craig \& Brown 1986; McIntosh 2000, and references therein) where solutions are not unique. However, improvements can be made with a careful selection of lines, and the larger uncertainties become those associated with either the observational data or the atomic physics calculations. Also note that the $D E M(T)$ does not necessarily have a direct physical significance (in terms of e.g. a temperature gradient), unless other factors are taken into account. For example, filling factors (cf. Judge 2000) are known to be small in the transition region, and probably different from those of the low corona.

Once the $D E M$ is derived from a set of observed fluxes, it is possible to calculate an emission measure value $E M_{\Delta T}\left(T_{i}\right)$ for each temperature $T_{i}$, once a temperature interval $\Delta T$ is defined:

$E M_{\Delta T}\left(T_{i}\right) \equiv \int_{T_{i}-\frac{\Delta T}{2}}^{T_{i}+\frac{\Delta T}{2}} \operatorname{DEM}(T) \mathrm{d} T$

Note that many different definitions of $D E M$ and $E M$ are found in the literature, that, together with some confusion in the terminology adopted, can create some difficulties when comparing results from different papers. For the sake of clarity, we therefore now briefly summarise other common definitions. In order to avoid the use of spherical symmetry and of the star's radius (a parameter not always well known), a volume differential emission measure $\operatorname{DEM}(T)=N_{\mathrm{e}} N_{\mathrm{H}} \frac{\mathrm{d} V}{\mathrm{~d} T}\left(\mathrm{~cm}^{-3} \mathrm{~K}^{-1}\right)$ and a corresponding emission measure $E M$ are often defined. The ionisation 
state is often assumed known by assigning a value to the ratios of the electron and hydrogen number densities (e.g. $N_{\mathrm{H}} / N_{\mathrm{e}} \simeq 0.8$ by assuming that $\mathrm{H}$ and $\mathrm{He}$ are fully ionised and $\left.A_{\mathrm{b}}(\mathrm{He})=0.1\right)$.

Another definition that is commonly used (see, e.g. Griffiths \& Jordan 1998, and references therein) involves differentiation over the logarithm of the temperature: $D E M_{\log }(T)=N_{\mathrm{e}} N_{\mathrm{H}} \frac{\mathrm{d} V}{\mathrm{~d} \log T}$. This definition is more convenient when an approximation for the volume emission measure is sought. In fact, if one defines an average temperature $T_{0}$ such as $\log T_{0}=$ $\int G(T) \log T \mathrm{~d} \log T / \int G(T) \mathrm{d} \log T$, and assumes that $D E M_{\log }(T)$ varies linearly with $\log T$, the total emission measure over an interval $\Delta \log T=0.3$ centred at $T_{0}$ can be approximated with what is generally termed the $\operatorname{EM}(0.3)$ value (cf. Griffiths \& Jordan 1998):

$$
E M_{\Delta \log T=0.3}\left(T_{0}\right) \simeq \operatorname{EM}(0.3) \equiv \frac{0.3 d^{2} I_{\mathrm{ob}}}{\int G(T) \mathrm{d} \log T}
$$

where $I_{\mathrm{ob}}$ is the observed flux at Earth. The $E M(0.3)$ points are often used instead of the $E M_{\Delta T}$ values. Note that in the definition of $E M(0.3)$ in Eq. (8) of Griffiths \& Jordan (1998) $F_{*}$ should read $4 \pi d^{2} F_{\text {obs }}$ according to their previous definitions. Also note that the $E M(0.3)$ values are generally plotted at the temperature $T_{\max }$ where $C(T)$ has a maximum, and not at $T_{0}$, as they should.

Another different and common approach (see Fig. 8) is to plot the ratio $I_{\mathrm{ob}} / G(T)$ for each line as a function of temperature and consider the loci of these curves to constrain the shape of the emission measure distribution. In fact, for each line and temperature $T_{i}$ the value $I_{\mathrm{ob}} / G\left(T_{i}\right)$ represents an upper limit to the value of the emission measure at that temperature (assuming that all the observed emission $I_{\mathrm{ob}}$ is produced by plasma at temperature $T_{i}$ ).

\subsection{Anomalous behaviour of $\mathrm{Li}$ - and $\mathrm{Na}$-like ions}

It should be noted that the ionisation equilibrium plays a major role not only in the derivation of the $D E M$, but also in that one of the elemental abundances.

The anomalous behaviour of the ions of the $\mathrm{Li}$ and $\mathrm{Na}$ isoelectronic sequences has been known for more than 30 years. For anomalous behaviour we mean that once a $D E M$ analysis is performed using lines from different isoelectronic sequences, the theoretical intensities of the lines of the anomalous ions are consistently under- or overestimated by large factors. Burton et al. (1971) reported this anomalous behaviour based on a solar spectrum from a rocket flight. Dupree (1972) confirmed this anomaly, using OSO-IV solar spectra. A possible explanation of this anomaly resides in the density dependence of the ionisation fractions. Burgess \& Summers (1969) showed that the density dependence of the dielectronic recombination, together with the collisional ionisations from metastable levels produce significant changes in the ionisation fractions. Vernazza \& Raymond (1978) took into account these effects and showed that significant increases in the line intensities of the Li-like ions occur at high densities. The same applies to the Na-like ions, and, to a lesser extent, to other ions as well. Raymond \& Doyle (1981a, 1981b) applied the ionisation equilibrium calculations of Vernazza \& Raymond (1978) to Skylab data and found a good agreement between lines of different isoelectronic sequences. The problem appeared to be resolved.

However, the same authors (Doyle \& Raymond 1984), among others, presented cases where these density effects were not enough to explain the anomalous behaviour. At that time, uncertainties in the atomic data and instrument's calibration were a major concern. Judge et al. (1995) presented a $D E M$ analysis of a rocket spectrum of the entire Sun, obtained with a good photometric accuracy of $15 \%$. They used more recent atomic data, and calculated the ionisation equilibrium taking into account the density effects. They found "very significant and systematic differences" between the line intensities of the $\mathrm{Li}$ and $\mathrm{Na}$ isoelectronic sequences and those of the other ions. $\mathrm{C}$ IV, $\mathrm{N} \mathrm{V}$, and $\mathrm{O}$ VI lines were underestimated by factors of 2 to 5 . Regarding the Na sequence, only Si IV was observed, with an underestimation by a factor of 2 .

Another difficulty in investigating these effects was due to the fact that the majority of the strong lines that could be used for $D E M$ analyses were from the anomalous class. For example, Raymond \& Doyle (1981b) used Li-like lines (O VI, Ne VIII, Mg X, Si XII, S XIV), with the exception of Ar VII and Ca X, to constrain the million-degree peak of the DEM. The same argument applies to the Judge et al. (1995) results.

The problems of the paucity of the observed lines and uncertainty in the instrument's calibration have been overcomed with the spectroscopic instruments on board SOHO. Del Zanna (1999) has presented many DEM analyses of different solar regions, using SOHO/Coronal Diagnostic Spectrometer (CDS) spectra. The CDS instrument covers almost entirely the $150-800 \AA$ spectral region, and is rich in emission lines from a large number of highly ionised ions of many different isoelectronic sequences. The CDS instrument has a good in-flight calibration (see Del Zanna et al. 2001a and references therein). The lines from the following ions have presented significant deviations (factors of 2 to 10): Ne VIII, Na IX, Mg X, Al XI, Si XII (Li-like); Ca X (Na-like). Del Zanna (1999) also found similar deviations with re-analyses of solar spectra from various instruments (including SERTS, Skylab). These discrepancies cannot be ascribed to elemental abundance anomalies, nor to instrumental effects.

Furthermore, progress in the theoretical models for ionisation and recombination processes has led to significant changes in the ionisation and recombination rates over the years. The compilation of Mazzotta et al. (1998) is the best available to date, computed for the low density limit. The Mazzotta et al. results have been used here. They are close to those of Arnaud \& Raymond (1992) for the Fe ions. For the other elements the differences from the older calculations of Arnaud \& Rothenflug (1985) are quite substantial, as discussed by Del Zanna (1999). Del Zanna et al. (2001b) have reanalysed Skylab data 
using the Mazzotta et al. (1998) ionisation fractions and up-to-date atomic data to show the extent of the anomalous behavior. The results are that lines of the Li-like $\mathrm{N} \mathrm{V}$ and C IV are underestimated by factors of 3 and 10, while those of Ne VIII and Mg X are overestimated by factors of 5 and 10, respectively. The S VI 933.3 $\AA$ (Na-like) is also underestimated by a factor of 3 .

We conclude that all the spectral lines of the $\mathrm{Li}$ and $\mathrm{Na}$ isoelectronic sequences observed in EUV solar spectra present significant deviations from other isoelectronic sequences. The analyses presented in Del Zanna (1999) also indicate a new characteristic. All lines in the lower transition region (e.g. C IV) are consistently underestimated, while those in the upper transition region (e.g. Ne VIII) are consistently overestimated. The deviations vary, depending on the source region observed. This suggests to us that one of the main inaccuracy in the ion balance calculations is the temperature corresponding to the peak emission. Until the reasons for these discrepancies are fully understood, and all the relevant physical effects taken into account, the lines of the $\mathrm{Li}$ and $\mathrm{Na}$ isoelectronic sequences should not be used for $D E M$, density and elemental abundance analyses.

\subsection{Elemental abundances}

Stellar coronal abundances are still poorly known, but are important to measure, because they affect our understanding of the stellar atmospheres. Comparisons with the solar case are difficult, since a large variety of solar coronal abundances have been reported, with variations from the photospheric values up to an order of magnitude (cf. the reviews of Feldman 1992; Raymond et al. 2001). These differences appear to be related to the first ionisation potential (FIP) of the various elements. The abundances of elements with low FIP $(<10 \mathrm{eV}$, e.g. Fe) appear enhanced compared to those of the high FIP ( $>10 \mathrm{eV}$, e.g. Ne) elements, using the solar photospheric values as a standard reference. Sulfur is an important element since it lies in the middle of these two classes. This "FIP effect" can be used as an important diagnostic of the physical processes that occur in the chromosphere and TR of active stars.

In principle, it is possible to derive a relative element abundance $A_{\mathrm{b}}\left(X_{1}\right) / A_{\mathrm{b}}\left(X_{2}\right)$ of two elements $X_{1}$ and $X_{2}$ from any observed intensity ratio $I_{1} / I_{2}$ :

$$
\frac{A_{\mathrm{b}}\left(X_{1}\right)}{A_{\mathrm{b}}\left(X_{2}\right)}=\frac{I_{1} \times \int C_{2}\left(T, N_{\mathrm{e}}\right) D E M(T) \mathrm{d} T}{I_{2} \times \int C_{1}\left(T, N_{\mathrm{e}}\right) D E M(T) \mathrm{d} T} .
$$

In practice, the ionisation balance, the selection of lines, and the spectroscopic method used can each account for a variation of a factor of two or more in the derived element abundances. Various examples are given in Del Zanna (1999) and Del Zanna et al. (2001b).

In the investigation of elemental abundances and the FIP effect it has been common to use ratios of low- vs. high-FIP lines that are emitted over the same temperature range and have similar contribution functions. In the solar case lines of Mg VI and Ne VI have been widely used. In the FUSE spectra the following ions present some temperature overlap: S III, S IV vs. N III; C III vs. N III; Si III vs. S III and C III; Ne VI vs. O VI; Ne V vs. S VI. The FUSE observations are complementary to those of Chandra and XMM, which observe coronal ions.

In the literature it is common to assume that any intensity ratio is directly proportional to the relative abundances of the two elements involved. A common approach, proposed by Widing \& Feldman (1989), is to approximate Eq. (4) by defining for each spectral line a single differential emission measure $D E M_{\mathrm{L}}$ value for the temperature range over which the line forms:

$D E M_{\mathrm{L}} \equiv\left\langle N_{\mathrm{e}} N_{\mathrm{H}} \frac{\mathrm{d} h}{\mathrm{~d} T}\right\rangle \equiv \frac{I_{\mathrm{ob}}}{A_{\mathrm{b}}(X) \int C(T) \mathrm{d} T}$.

The relative elemental abundances are then adjusted in order to make the $A_{\mathrm{b}}(X) D E M_{\mathrm{L}}=I_{\mathrm{ob}} / \int C(T) \mathrm{d} T$ points lie along a common smooth curve. These points are displayed at the temperatures $T_{\max }$, defined as the temperature where $C(T)$ has a maximum. This method can be misleading. First, the use of $T_{\max }$ is problematic, since this temperature can in some cases be quite different from the temperature where the bulk of the plasma is emitted. An effective temperature $T_{\text {eff }}$, defined as $\log T_{\text {eff }}=$ $\int C(T) \operatorname{DEM}(T) \log T \mathrm{~d} T /\left(\int C(T) D E M(T) \mathrm{d} T\right)$ is more appropriate. Second, this method neglects the shape of the $D E M$ distribution (which is not known a priori) and can therefore lead to a large under- or over-estimation of the relative abundance. This can occur if for example the $\operatorname{DEM}(T)$ has a steep gradient in the temperature range where $C_{1}(T)$ and $C_{2}(T)$ have some differences (see Del Zanna et al. 2001b for details).

The approach adopted here is to use the largest possible number of lines, calculate the line emissivities at the measured TR densities, and then determine the relative element abundances and the $\operatorname{DEM}(T)$ at the same time. The scaling between the high and low-FIP elements is done using lines that overlap in temperature. More details of the technique can be found in Del Zanna \& Bromage (1999). In this way, most of the uncertainties will be reduced, and any systematic effects, such as those occurring with the Na- and Li-like lines will be highlighted. The use of line ratio methods for abundance diagnostics of solar and stellar coronae, in particular when lines of the $\mathrm{Na}$ and $\mathrm{Li}$ isoelectronic sequences are used, as recently suggested by e.g. Laming \& Feldman (1999), is inadvisable.

\subsection{Electron density}

The most accurate density diagnostics are those obtained by considering the ratios of lines emitted by the same ion which are sensitive to density. However, owing to the paucity of the observed density-sensitive line pairs from the same ion, other methods have often been adopted in solar and stellar physics. One method is to compare the emission measures of the forbidden lines with those of the allowed ones. One of the first applications of this 
method was due to Feldman et al. (1977). A variation of this method is to compare the emission measure loci of the two groups of lines, and is the most commonly used (see, e.g., Brown et al. 1984b; Byrne et al. 1987; Jordan et al. 1987; Linsky et al. 1989; Maran et al. 1994; Linsky et al. 1995; Griffiths \& Jordan 1998; Pagano et al. 2000). In most cases, large inconsistencies between the densities obtained using this method with those derived from line ratios have been reported. These inconsistencies are caused by the use of lines with anomalous behaviour, as shown below in Sect. 5.3.

Diagnostics based on line ratios have been extensively used in solar and stellar physics. In many cases, discrepancies between densities derived using different line ratios or different ions have been reported, thus leading to suggestions that the derived values might not have any physical significance (Judge \& McIntosh 1999). It is well known that significant effects on the line ratios can be caused by inhomogeneities in the atmosphere (see e.g., Doschek 1984) or by plasma flows (cf. Raymond \& Dupree 1978). However, we would like to stress that some of these discrepancies could also be due to un-accounted blending and/or inaccurate atomic calculations, as shown below in Sect. 5.2 with some examples. In addition, that misleading results can easily be obtained by using line ratios that have a very small sensitivity at the observed densities or when temperature effects are not taken into account. In some cases, it is possible to obtain consistent measurements, although it should be born in mind that the derived densities are in any case averaged values over the entire stellar disk and along the line of sight.

Finally, it is common in the literature to use only a few well-behaved line ratios, without considering all the observed lines. A different approach, preferable when more than two lines from an ion are observed, is to plot the values of

$f_{j i}=\frac{I\left(\lambda_{i j}\right) N_{\mathrm{e}}}{h \nu_{i j} N_{j}\left(N_{\mathrm{e}}\right) A_{j i}}$

as a function of electron density, calculated at a fixed temperature. All the curves should cross at one point, if the plasma is isodensity (a description of this method, termed $L$-function, can be found in Landi \& Landini 1998). The $f_{j i}$ curves should be calculated at the effective temperature, i.e. at the temperature where the bulk of the ion emission is. This can be quite substantially different from the temperature of the maximum ionisation fraction, in particular when the $D E M$ has a steep gradient at the temperatures where the ion is emitted. This method has the following advantages over the line ratio technique: a) it gives an overall view of all lines; b) it shows any discrepancies even when considering lines that are not densitysensitive; c) it clearly shows which lines (and not ratios) are more suitable in a particular density regime; d) it clearly shows when lines are at the limit of their density sensitive regime. We use this method in Sect. 5.2 to discuss individual cases.

\section{AU Mic multi-wavelength observations}

The value of using multi-wavelength observations is evident from the above. Unfortunately it is difficult to obtain simultaneous observations with different satellites, and most authors merge observations that can be even years apart. This procedure has some limitations, since active stars are known to present high variability in the $\mathrm{XUV}$ on all time scales, from minutes (due to flaring) to years (possibly due to stellar cycles). In this paper we consider FUSE, STIS and EUVE observations of the dMe star AU Mic as a benchmark study. AU Mic is a nearby $(9.94 \mathrm{pc})$ dM1e star smaller than the Sun $\left(0.59 R_{\odot}\right)$. We have selected AU Mic for two main reasons.

The first is that AU Mic is one of the brightest ultraviolet and X-ray sources, observed by several satellites. EUVE observed AU Mic in quiescent and flaring state on July 14-17, 1992. A detailed analysis of the main plasma parameters during both phases was presented by Monsignori Fossi et al. (1996). AU Mic was also observed on July 22-23, 1993 (Del Zanna et al. 1995) during a quiet phase, with count rates very similar to those measured during the quiescent phase of the July 1992 observation. We have therefore adopted the July 1993 EUVE observation as representative of AU Mic in quiescence. STIS observed AU Mic on September 6, 1998 in both quiescent and flaring state. An analysis of the quiescent part of this observation (totaling to 9200s) has been presented by Pagano et al. (2000), while Robinson et al. (2001) discusses the flaring part.

The second reason is that the spectroscopic diagnostics that have been applied to AU Mic by other authors present some of the problems that we highlight in this paper. $D E M$ analyses and calculations of radiative losses for AU Mic in quiescence have been presented by Quin et al. (1993, using IUE spectra) and by Pagano et al. (2000). The latter measured densities using different methods and ions and found some of the commonly known inconsistencies. In particular, Pagano et al. found a quite substantial discrepancy between the density derived from O IV ratios $\left(\log N_{\mathrm{e}} \simeq 10.8\right)$ and that deduced with the emission measure loci method $\left(\log N_{\mathrm{e}}=11.5-12\right)$. We use a new FUSE observation of AU Mic in order to complement those of STIS and EUVE, and revise the results of Quin et al. and Pagano et al. in terms of densities, DEM and elemental abundances.

\subsection{FUSE observations of AU Mic}

AU Mic was observed in August 2000 with the FUSE large square aperture (LWRS: $30^{\prime \prime} \times 30^{\prime \prime}$ ), starting at 19:35:47 UT on the 26th and ending at 09:21:52 UT on the $27 \mathrm{th}$, with a total of 9 observations (one for each orbit) of about $30 \mathrm{~min}$ each. We have used the publicly available data, processed with the standard FUSE pipeline package (CALFUSE version 1.7.7). Among all the channels, the $\mathrm{SiC} 2 \mathrm{~A}, \mathrm{LiF} 1 \mathrm{~A}$, and $\mathrm{LiF} 2 \mathrm{~A}$ present the highest effective areas and are those that have been considered here. 


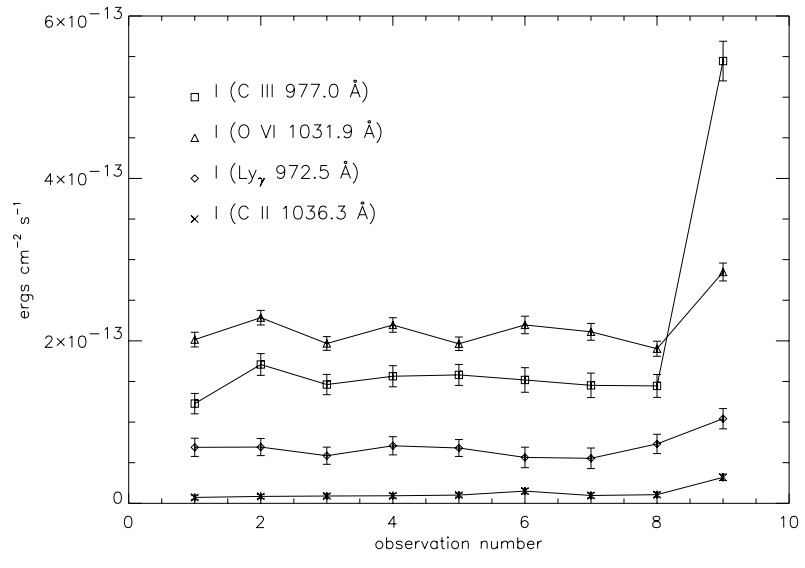

Fig. 1. Fluxes of a selection of lines observed by FUSE during the AU Mic observation. Note the presence of a flare during the last observation.

The standard wavelength calibration required some corrections. Normally, the wavelength scale for a given observation has a variable zero point offset. The single observations did not require any wavelength adjustments. On the other hand, large wavelength shifts of $-0.25,-0.3$, and $-0.15 \AA$ were required to adjust the calibration of the $\mathrm{SiC} 2 \mathrm{~A}, \mathrm{LiF} 1 \mathrm{~A}$, and $\mathrm{LiF} 2 \mathrm{~A}$ channels, respectively (similar to what found by others, see, e.g., Harper et al. 2001). These shifts produce a good representation of the lines observed, as can be seen from a comparison with the theoretical wavelengths obtained from the CHIANTI database, and shown in Table 3 (note that the theoretical wavelengths are given with three decimal places for easier comparison with the observations, although they are not always known with such accuracy).

First, an analysis of the 9 observations has been performed. The $S / N$ is such that only the fluxes of the brightest lines could be measured, for each observation. Figure 1 shows the time variation of the fluxes of a selection of lines during the 9 observations. The star was obviously in a quiescent state during the first 8 orbits, while during the last observation a large flare occurred. All the transition region lines increased their intensities by factors of 2 to 5 . The C III, Si IV lines presented the largest increase, while lines emitted at lower (e.g. C II, Ly $y_{\gamma}$ ) or higher (e.g. O VI) temperatures were only slightly affected. Lines emitted at coronal temperatures (Fe XVIII, Fe XIX) did not show any significant enhancement. This event cannot be classified as a classical stellar flare, but instead as a transition region explosive event. The paper by Robinson et al. (2001) focuses on brightenings with similar characteristics, although of much smaller amplitude. Something similar was also observed with STIS by Ayres (2001) during a multi-wavelength (EUVE, Chandra, STIS) campaign on the RS CVn binary HR 1099. It is possible that these types of stellar transition region events are very common in active stars. The lower sensitivity of previous instruments only allowed the observation of the long duration flares.
Aside from the flare, all the previous 8 observations present a remarkable constancy in fluxes. We therefore proceeded by averaging the first 8 spectra of each channel, for a total of $15686 \mathrm{~s}$. In order to further increase the $S / N$, the spectra have also been rebinned. The line fluxes have been measured with multiple profile fitting and removal of the background. The errors have been calculated according to the $S / N$ in the line, and derived directly from the extracted spectra in counts. The results are shown in Table 3.

Taking into account the relatively short exposure time, a considerable number of lines are clearly detected (see Fig. 2). All lines are formed in the chromosphere and transition region, with the exception of two coronal lines. In fact, we can positively identify not only the Fe XVIII $974.86 \AA$ line (already observed in the Capella spectrum by Young et al. 2001), but also the bright Fe XIX 1118.06 $\AA$ line. The blending of C I lines with the Fe XIX line can be excluded, since C I lines are very weak in the spectrum. The identification of the Fe XIX line as well as the others is further confirmed by the $D E M$ analysis (see Sect. 5.3 and Table 3).

\subsection{Merging of the datasets}

First, we have verified that the fluxes in the TR lines observed by STIS and FUSE during quiescence are consistent. In particular, the fluxes of the C III multiplet at $1175 \AA$ as measured by both instruments are similar, with a deviation of only $7 \%$, well within the statistical and calibration errors. Second, it is interesting to note that the fluxes of the TR lines observed by STIS and FUSE during quiescence are consistent with those obtained by IUE (Linsky et al. 1982; Ayres et al. 1983; Butler et al. 1987; Quin et al. 1993). This gives us confidence in the merging of the two datasets, that should be representative of the $\mathrm{AU}$ Mic quiescent "average transition region". We have used the line fluxes of Pagano et al. (2000), assuming for the $D E M$ analysis an indicative $20 \%$ error, since no error estimates were presented.

In this paper, we are mainly interested in the diagnostics applied to the transition region. However, we have complemented the STIS and FUSE data with the EUVE ones. We have verified that the Fe XXI $1354 \AA$ emission observed by STIS is consistent with the Fe XXI emission measured by EUVE, and that the EUVE fluxes are broadly consistent with the FUSE data (see Sect. 5.3), thus giving us confidence in the similarity of the coronal activity during these observations years apart.

\section{Results}

It is important to check that the observed lines are optically thin, before performing any density estimate or emission measure modeling. 

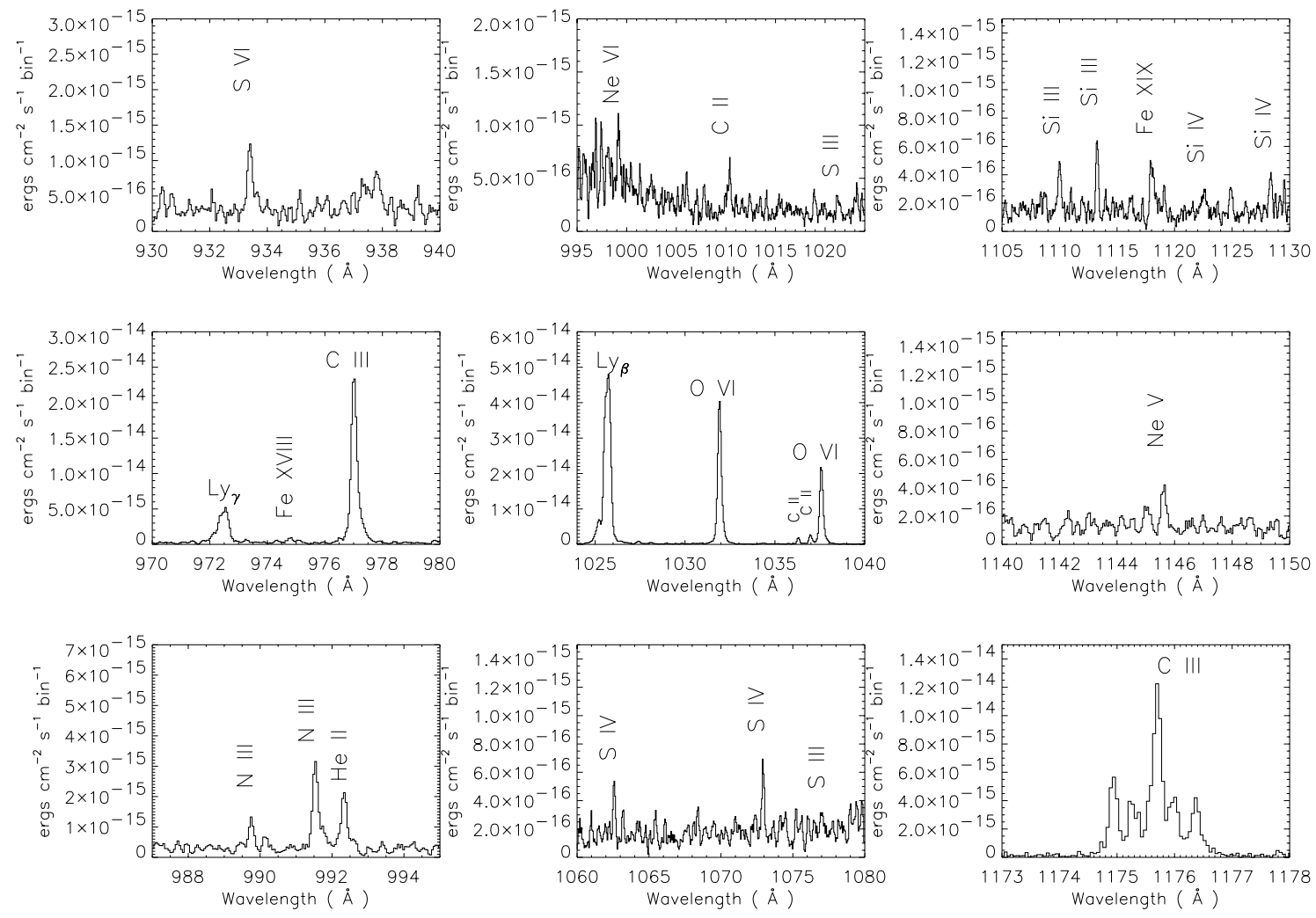

Fig. 2. A selection of FUSE wavelength ranges, with the AU Mic observed spectra.

\subsection{Optical depth}

Fortunately, it is possible in a number of cases to check which lines are optically thick, and to what extent. We can identify two groups of lines in the AU Mic STIS and FUSE observations (see Table 1). The first are the doublets of Si IV, C IV, N V, and O VI. In the optically thin case, the ratios of the two lines of the doublet should be equal to the ratio of the oscillator strengths, i.e. 2. If opacity effects are present, the ratio would decrease, with the brightest component ${ }^{2} \mathrm{~S}_{1 / 2}{ }^{2} \mathrm{P}_{3 / 2}$ being more affected, having a higher oscillator strength. In this case, the ratio is a direct measure of the ratio of the photon escape probabilities, from which the optical depths of the lines can be obtained (see e.g. Mathioudakis et al. 1999). The observed C IV, N V and O VI ratios indicate that the lines are optically thin. Only Si IV shows an opacity effect, with the ${ }^{2} \mathrm{~S}_{1 / 2}{ }^{2} \mathrm{P}_{1 / 2}$ line having an optical depth of about 0.3 , corresponding to a very small correction factor (a 15\% increase).

The other groups of lines that can be used to estimate the opacity are those where there are ratios of lines that originate from a common upper level (a branching ratio). In the optically thin case, the ratios are equal to the ratios of the $A$-values (see, e.g. Jordan 1967). The STIS and FUSE spectra contain a number of useful ratios, of C II, Si II, Si III, and C III. The C II ratio indicates the presence of opacity effects, although of smaller amplitudes than those reported on the Sun (see, e.g. Brooks et al. 2000). Also Si II appears to be affected by opacity. The Si III is the worst case, since the $1296.726 \AA$ line
Table 1. The line ratios in the STIS and FUSE spectral ranges useful for optical depth estimates. The last two columns indicate the theoretical (optical thin case) and the observed ratio values.

\begin{tabular}{|c|c|c|c|c|}
\hline Ion & Terms & Wavelengths $(\AA)$ & Th. & $\mathrm{Ob}$ \\
\hline \multirow[t]{2}{*}{ C II } & ${ }^{2} \mathrm{P}_{3 / 2}{ }^{-2} \mathrm{~S}_{1 / 2} /$ & & & \\
\hline & ${ }^{2} \mathrm{P}_{1 / 2}-{ }^{2} \mathrm{~S}_{1 / 2}$ & $1037.020 / 1036.332$ & 2 & 1.5 \\
\hline \multirow{3}{*}{$\begin{array}{l}\text { Si II } \\
\text { C III }\end{array}$} & $\prime \prime$ & $1533.430 / 1526.706$ & 2 & 1.2 \\
\hline & ${ }^{3} \mathrm{P}_{0}{ }^{-3} \mathrm{P}_{1} /$ & & & \\
\hline & ${ }^{3} \mathrm{P}_{2}-{ }^{3} \mathrm{P}_{1}$ & $1175.265 / 1176.372$ & 0.8 & 1.0 \\
\hline \multirow{3}{*}{$\begin{array}{l}\text { Si III } \\
\text { C III }\end{array}$} & $\prime \prime$ & $1296.726 / 1303.323$ & 0.8 & 0.5 \\
\hline & ${ }^{3} \mathrm{P}_{2}-{ }^{3} \mathrm{P}_{2} /$ & & & \\
\hline & ${ }^{3} \mathrm{P}_{1}-{ }^{3} \mathrm{P}_{2}$ & $1175.713 / 1174.935$ & 3 & 1.9 \\
\hline \multirow{3}{*}{$\begin{array}{l}\text { Si III } \\
\text { Si IV }\end{array}$} & "I & $1298.944 / 1294.543$ & 3 & 2 \\
\hline & ${ }^{2} \mathrm{~S}_{1 / 2}{ }^{-{ }^{2}} \mathrm{P}_{3 / 2} /$ & & & \\
\hline & ${ }^{2} \mathrm{~S}_{1 / 2}{ }^{-2} \mathrm{P}_{1 / 2}$ & $1393.755 / 1402.770$ & 2 & 1.7 \\
\hline C IV & "I & $1548.201 / 1550.772$ & 2 & 2 \\
\hline N V & $\prime \prime$ & $1238.821 / 1242.804$ & 2 & 2 \\
\hline $\mathrm{O} \mathrm{VI}$ & $\prime \prime$ & $1031.914 / 1037.615$ & 2 & 2 \\
\hline
\end{tabular}

is very weak in the spectrum, and the $1298.944 \AA$ line is blended with another line of the multiplet. If the blend is accounted for, the observed ratio indicates some opacity in the $1298.944 \AA$ line, similarly to the solar case (cf. Keenan \& Kingston 1986). 
The first C III ratio is consistent with the theoretical value, while the second one is lower than the predicted one, suggesting some opacity. The latter result is more uncertain, because the measurement of the $1175.713 \AA$ line is difficult, owing to the presence of the $1175.592 \AA$ line. Even with the high spectral resolution of FUSE these two lines are difficult to deblend with a multiple profile fitting. In any case, these C III results are similar to those obtained from solar observations (see, e.g., Doyle \& McWhirter 1980; Brooks et al. 2000) and indicate that within the multiplet the $1175.713 \AA$ line has the highest optical thickness, as expected. It is worth noting that the Si III and C III line ratios can be affected by any inhomogeneities of the atmosphere (see, e.g. Doschek \& Feldman 1977) because of their density and temperature sensitivity.

In conclusion, we can say that only a small number of lines are somewhat affected by opacity. However, no corrections to the line fluxes have been applied, since they would only slightly affect the lines at the lower temperatures and would not change the main results discussed in the $D E M$ analysis (Sect. 5.3).

\subsection{Electron density}

In what follows, we review the density diagnostics relevant to active stars in the STIS and FUSE spectral regions, by comparing observations with the best atomic data available.

\subsubsection{O IV and S IV}

The O IV and S IV lines around $1400 \AA$ (see Table 2) are well known density diagnostics in both solar and stellar spectra (cf. Linsky et al. 1995; Cook et al. 1995; Brage et al. 1996; Harper et al. 1999; Teriaca et al. 2001, and references therein). They are considered to be the best diagnostics in the FUV wavelength range because their temperature sensitivity is very small, compared to other cases such as C III, O V and Si III (see below). However, a number of problems and inconsistencies have been reported in the literature, leading to a degree of confusion on the reliability of the O IV and S IV lines. In what follows, we will discuss several issues, complementing the AU Mic observations with those of Cook et al. (1995) and Linsky et al. (1995). Cook et al. (1995) presented a comprehensive list of O IV and S IV solar observations (including active regions, flares), while Linsky et al. (1995) published one of the best spectrum in the FUV, based on HST/GHRS observations of Capella.

Let us consider O IV first. The $1397.217 \AA$ is very weak and is rarely observed even in solar spectra. The 1399.779 $\AA$ line is also very weak, and usually has a large observational error associated with it. The 1399.779/ 1407.383 $\AA$ is a branching ratio, and excellent agreement between theory and observations is normally found (e.g. Cook et al. 1995). The ratios that include the $1401.171 \AA$ line $\left(R_{1}, R_{2}, R_{3}\right)$ have often been used to estimate the
Table 2. The O IV and S IV transitions in the STIS spectral range and some of the density-sensitive ratios commonly used.

\begin{tabular}{lcll}
\hline \hline Ion & Terms & Wavelength $(\AA)$ & Ratio \\
\hline O IV & ${ }^{2} \mathrm{P}_{1 / 2}{ }^{-}{ }^{4} \mathrm{P}_{3 / 2}$ & 1397.217 & \\
O IV & ${ }^{2} \mathrm{P}_{1 / 2}-{ }^{4} \mathrm{P}_{1 / 2}$ & 1399.779 & \\
O IV & ${ }^{2} \mathrm{P}_{3 / 2}{ }^{-} \mathrm{P}_{5 / 2}$ & 1401.171 & \\
O IV & ${ }^{2} \mathrm{P}_{3 / 2}-{ }^{4} \mathrm{P}_{3 / 2}$ & $1404.793(\mathrm{bl})$ & \\
O IV & ${ }^{2} \mathrm{P}_{3 / 2}-{ }^{-} \mathrm{P}_{1 / 2}$ & 1407.383 & \\
O IV & & $1401.171 / 1399.779$ & $R_{1}$ \\
O IV & & $1401.171 / 1407.383$ & $R_{2}$ \\
O IV & & $1401.171 / 1404.793$ & $R_{3}$ \\
O IV & & $1404.793 / 1407.383$ & $R_{4}$ \\
O IV & & $1404.793 / 1399.779$ & $R_{5}$ \\
S IV & ${ }^{2} \mathrm{P}_{1 / 2}-{ }^{4} \mathrm{P}_{3 / 2}$ & 1398.040 & \\
S IV & ${ }^{2} \mathrm{P}_{1 / 2}{ }^{-} \mathrm{P}_{1 / 2}$ & $1404.808(\mathrm{bl})$ & \\
S IV & ${ }^{2} \mathrm{P}_{3 / 2}{ }^{-} \mathrm{P}_{5 / 2}$ & 1406.016 & \\
S IV & ${ }^{2} \mathrm{P}_{3 / 2}{ }^{-}{ }^{4} \mathrm{P}_{3 / 2}$ & 1416.887 & \\
S IV & ${ }^{2} \mathrm{P}_{3 / 2}{ }^{-} \mathrm{P}_{1 / 2}$ & 1423.839 & \\
S IV & & $1416.887 / 1406.016$ & $R_{6}$ \\
S IV & & $1423.839 / 1416.887$ & $R_{7}$ \\
\hline
\end{tabular}

density, even though they have a very small density sensitivity as already noted by Feldman \& Doschek (1979) and as can be judged from Fig. 3. Even with accurate measurements, large errors should be expected, particularly for densities higher than $10^{10} \mathrm{~cm}^{-3}$. On the other hand, ratios including the $1404.793 \AA$ line $\left(R_{4}, R_{5}\right)$ have a much better density sensitivity, and have therefore often been preferred. Unfortunately, the $1404.793 \AA$ line is a wellknown blend with S IV (1404.808 ^) and with another unknown line (as described below), and therefore its use cannot preclude an understanding of the S IV emission.

The S IV $1416.887 \AA$ and $1423.839 \AA$ lines are weak and are often not detected, even in solar spectra. The 1398.040 $\AA$ line is even weaker. It is common practice in both solar and stellar physics to use the theoretical ratio of the $1404.808 \AA$ and $1406.016 \AA$ lines to infer the contribution of the $1404.808 \AA$ line to the observed blend with O IV. The first problem with this method is that the 1404.808/1406.016 $\AA$ ratio is density sensitive, and therefore, unless there is an independent way of measuring the density, it should not be used. The second problem is in explaining the S IV emission, as discussed below.

In previous publications, the S IV atomic calculations by Dufton et al. (1982) have normally been used. These calculations predict that the intensity of the $1404.808 \AA$ line is about 0.2 the intensity of the $1406.016 \AA$ line, up to densities of $3 \times 10^{11} \mathrm{~cm}^{-3}$. This results in a contribution to the observed blend that is normally only of the order of $5-10 \%$, i.e. it is generally assumed that the observed blend is mostly O IV $1404.793 \AA$. Now, a further problem is that many authors report inconsistencies between the densities obtained by using different line ratios. For 
example, Cook et al. (1995) report that densities derived from the $R_{4}$ ratio are consistently more than an order of magnitude smaller than those derived from the $R_{2}$ ratio, for a wide range of cases (Quiet Sun, Active Regions, Sunspots, Flares). Similarly, many authors found inconsistencies with the densities derived from S IV lines. For example, Cook et al. (1995) pointed out that the densities derived from the $R_{6}, R_{7}$ ratios are beyond the lowor high-density limit, and no comparisons with the densities derived from O IV could be made. These inconsistencies in the O IV and S IV lines therefore suggest various possibilities:

1. the atomic data for O IV are inaccurate;

2. the atomic data of Dufton et al. for S IV are inaccurate;

3. the blend observed at $1404.8 \AA$ is mostly due to an unidentified line, and not to either O IV or S IV or a combination of the two;

among with other more subtle explanations, such as fluorescence, suggested by Cook et al. (1995). Regarding option (1), we note that Cook et al. (1995) used the collisional $R$-matrix calculations of Zhang et al. (1994) for O IV. Brage et al. (1996) presented new O IV atomic structure calculations. They compared their O IV model with a wide range of solar and stellar observations, including the same data presented by Cook et al. (1995), and removing the S IV contribution to the $1404.8 \AA$ blend according to the Dufton et al. (1982) calculations. Brage et al. (1996) claimed that in most cases no inconsistencies were found, in contrast to previous studies such as in Cook et al. (1995). However, it should be noted that Brage et al. considered different line ratios $\left(R_{2}, R_{3}, R_{5}\right)$, and their results are therefore not directly comparable with those of Cook et al. (1995). We used the CHIANTI v3.02 atomic model for O IV, which includes the collisional $R$-matrix calculations of Zhang et al. (1994), together with $A$-values from Flower \& Nussbaumer (1975). The model gives results that are very close to those of Brage et al. (1996), as shown by Teriaca et al. (2001).

Regarding option (2), there is now substantial evidence for the inaccuracy of the Dufton et al. calculations. Aside from the studies already mentioned, Harper et al. (1999) used the same S IV ratios and atomic data of Cook et al. and found inconsistencies with the HST/GHRS spectra of the RR Tel nebula. Doschek et al. (1999) reported SOHO/SUMER measurements of S IV over a wide wavelength range (661-1406 $\AA$ ), and found very large disagreements with the Dufton et al. calculations, of up to a factor of 6 . We used the CHIANTI version 3.02 model for $\mathrm{S} I V$, that includes the recent $R$-matrix calculations of Tayal (2000). We have considered a large number of published solar and stellar observations, and found that the new atomic data for S IV gives very different results, in most cases in good agreement with the observations. A detailed discussion is beyond the scope of this paper. Here, we only mention that in the $661-900 \AA$ range the agreement with the Doschek et al. (1999) data is now excellent, and provide two examples to illustrate this point.
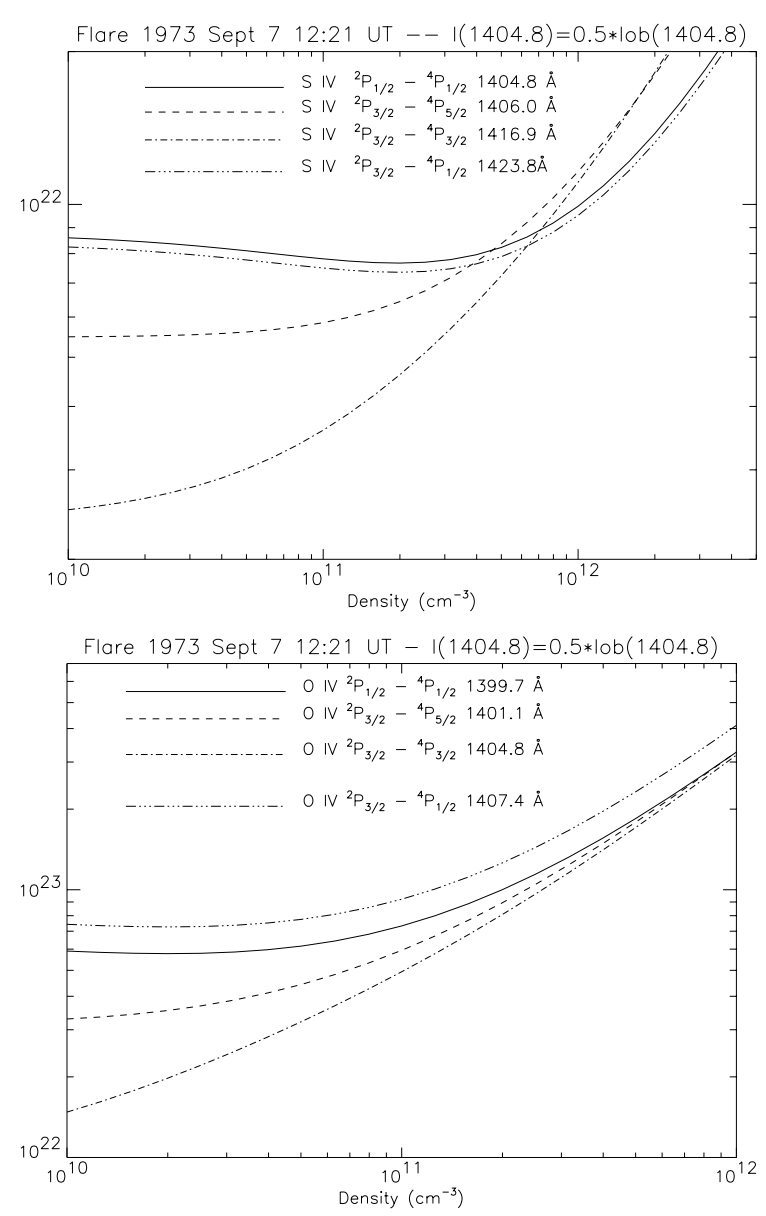

Fig. 3. The $f_{j i}$ curves of the S IV and O IV lines observed by HRTS I during a solar flare - 1973 Sep. 7 12:21 UT (Cook et al. 1995). We have assumed here that $50 \%$ of the intensity of the observed blend at $1404.8 \AA$ is due to the S IV $1404.808 \AA$ line, while the other $50 \%$ is due to O IV $1404.793 \AA$.

The first example is presented in Fig. 3, and shows the $f_{j i}$ curves of the O IV and S IV measurements of Cook et al. (1995) during a solar flare. Contrary to what Cook et al. found, it can be seen that the three unblended S IV lines do indicate a consistent density $\left(5 \times 10^{11} \mathrm{~cm}^{-3}\right)$. Agreement with the $1404.808 \AA$ line is also found by assuming that the S IV line contributes to $50 \%$ of the observed blend. If one assumes that the other $50 \%$ is all O IV 1404.793 $\AA$, agreement is also found with the other O IV lines, although all lines are in the high density limit and therefore no reliable density measurement can be established (contrary to what Cook et al. found). The problem with the O IV and S IV lines appears to have been resolved.

However, we found a number of cases in both solar and stellar spectra where additional blending in the $1404.8 \AA$ line seems to occur. Figure 4 shows the second example: the $f_{j i}$ curves of the O IV and S IV lines of one of the best stellar spectra (Capella - Linsky et al. 1995). The S IV $1404.808 \AA$ line is in agreement with the other S IV lines only if its intensity is $9 \%$ that of the observed line. If one assumes that the rest $(91 \%)$ is all due to O IV 

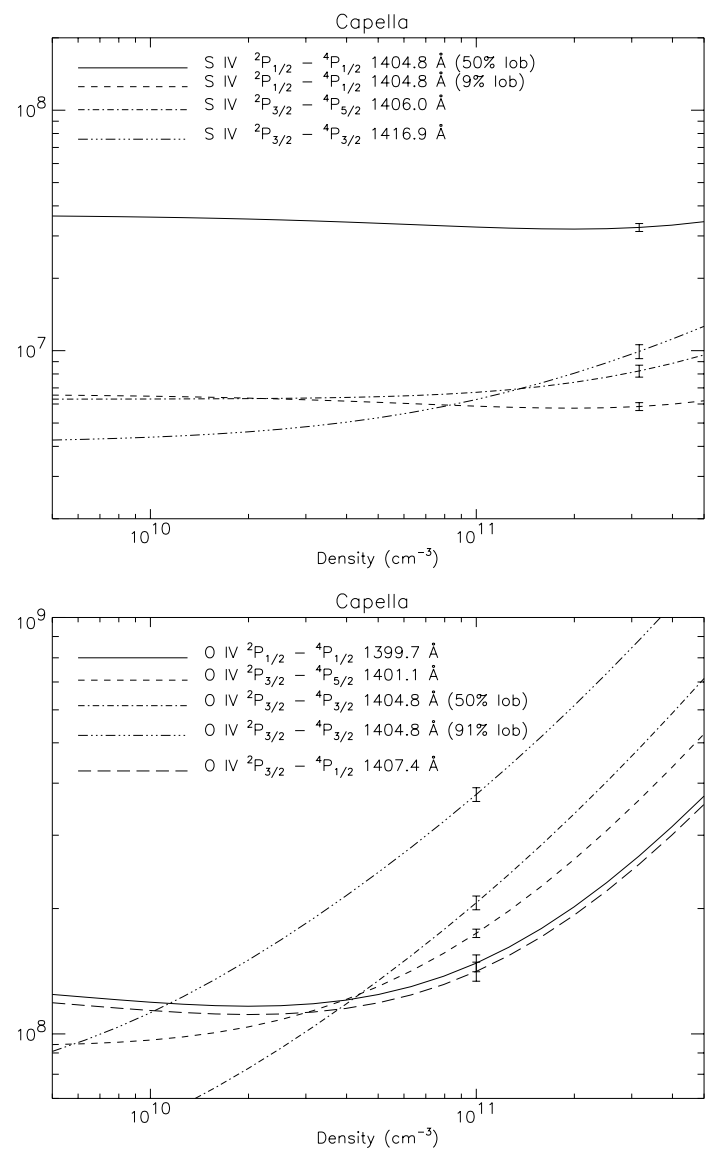

Fig. 4. The $f_{j i}$ curves of the S IV and O IV lines observed by HST/GHRS on Capella (Linsky et al. 1995).

1404.793 $\AA$, no agreement is found with the other O IV lines. Instead, the contribution of the O IV line should be about $50 \%$. This leaves about $40 \%$ of the observed line due to an unknown blend. The measurements have very small errors, indicated in the figure. Figure 4 also shows that no conclusive density measurement based on S IV can be obtained, while the O IV indicates a density of $4 \times 10^{10} \mathrm{~cm}^{-3}$, a value almost an order of magnitude higher than the value indicated by Linsky et al.

Let us now consider the FUSE and STIS AU Mic observations of S IV and O IV (see Fig. 5). To reach consistency with the other lines, the intensity of the S IV $1404.808 \AA$ line has been assumed equal to $20 \%$ the intensity of the observed blend. If we assume that the rest (80\%) of the observed blend is due to the O IV $1404.7 \AA$ line, we can see that the O IV $f_{j i}$ curves are consistent with a density of the order of $5-9 \times 10^{10} \mathrm{~cm}^{-3}$. The measurement of the $1399.779 \AA$ line is in obvious disagreement, and might be due to weakness of this line. The same holds for the S IV 1406.016 A line.

\subsubsection{O V}

The Be-like O V is particularly important for the STIS spectral range, since the ratio of the 1218.390 and $1371.292 \AA$ lines is an excellent diagnostic at the densi-
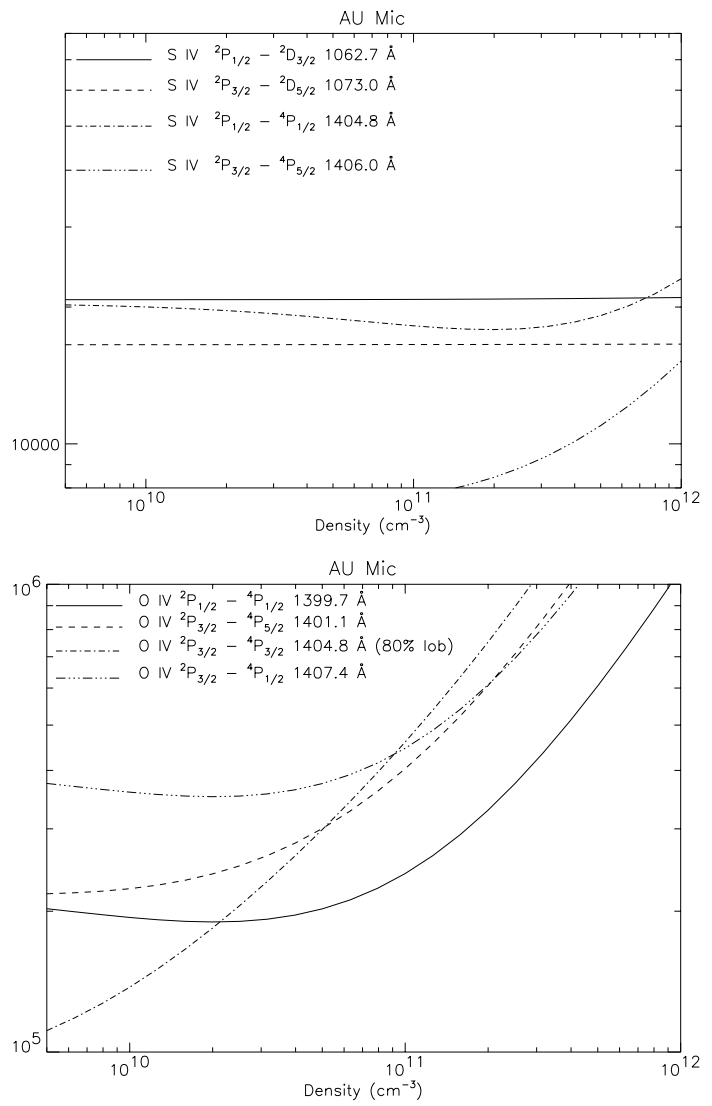

Fig. 5. The $f_{j i}$ curves of the S IV and O IV lines observed by STIS and FUSE. The intensity of the S IV $1404.808 \AA$ line has been assumed equal to $20 \%$ the intensity of the observed blend.

ties of active stars. However, many authors in the past reported discrepancies between the densities derived from this ratio with those obtained from other ions. For example, Pagano et al. (2000) used the Zhang \& Sampson (1992) collisional data and obtained from this ratio a density of $5 \times 10^{11} \mathrm{~cm}^{-3}$, at odds with what derived from the O IV ratios. If the $R$-Matrix calculations of Berrington et al. (1985) are used (CHIANTI version 3.02), large differences are found for some transitions, and the densities derived from $\mathrm{O} \mathrm{V}$ become consistent with those obtained from other ions, as shown in Fig. 6 . In fact, at the effective temperature $T_{\text {eff }}=2.7 \times 10^{5} \mathrm{~K}$ (see Table 3 ), the observed ratio indicates an electron density of $5 \times 10^{10} \mathrm{~cm}^{-3}$. Note that this $T_{\text {eff }}$ is quite different from the temperature of maximum ionisation fraction $\left(T=2.5 \times 10^{5} \mathrm{~K}\right.$ according to Mazzotta et al. 1998) and that a small difference in temperature has a large effect on the ratio (Fig. 6).

\subsubsection{Si III}

There are a few Si III lines visible in the FUSE and STIS spectral ranges that could be used for density diagnostics. For example, the resonance $3 \mathrm{~s}^{2}{ }^{1} \mathrm{~S}_{0}-3 \mathrm{~s} 3 \mathrm{p}{ }^{1} \mathrm{P}_{1}$ line at $1206.499 \AA$ has a density dependence different compared to the lines of the $3 \mathrm{~s} 3 \mathrm{p}^{3} \mathrm{P}-3 \mathrm{p}^{2}{ }^{3} \mathrm{P}$ multiplet. All the measurements are consistent with the density derived from 

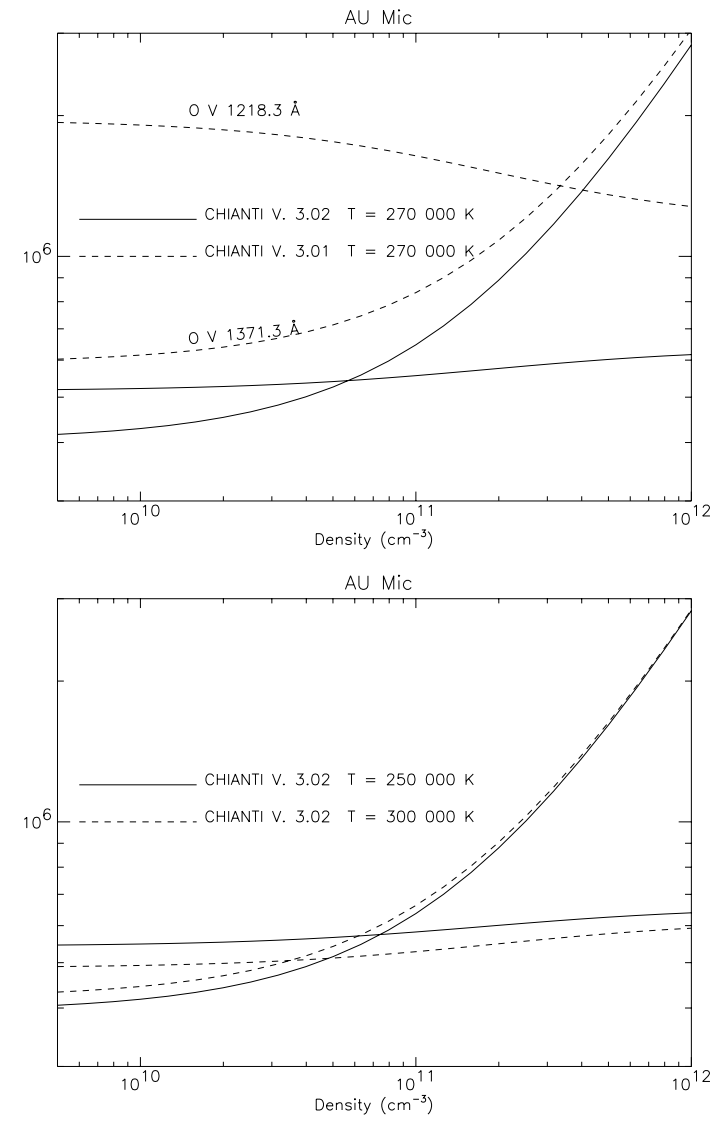

Fig. 6. The $f_{j i}$ curves of the O V lines observed in the STIS spectrum of AU Mic. Top: values calculated with the Zhang \& Sampson (1992 - CHIANTI v. 3.01) and Berrington et al. (1985 - CHIANTI v. 3.02) atomic data at the same temperature. Note the large difference. Bottom: values calculated at two temperatures. Note the large temperature effect.

O V, with two exceptions, the ${ }^{3} \mathrm{P}_{1}-{ }^{3} \mathrm{P}_{0}(1301.147 \AA)$ and the ${ }^{3} \mathrm{P}_{1}-{ }^{3} \mathrm{P}_{2}$ (1294.543 $\AA$ ) lines observed by STIS, which are probably blended. However, the Si III results have a large uncertainty, since the density dependence is small, and temperature effects are even greater than in the $\mathrm{O} \mathrm{V}$ case. This occurs because the DEM of AU Mic presents a a steep gradient at the Si III temperatures (see Sect. 5.3), larger than for $\mathrm{O} \mathrm{V}$.

\subsubsection{III}

Densities could be obtained using the well-known ratio of the resonance $2 \mathrm{~s}^{2}{ }^{1} \mathrm{~S}_{0}-2 \mathrm{~s} 2 \mathrm{p}{ }^{1} \mathrm{P}_{1}$ line at $977.022 \AA$ with the total intensity of the $2 \mathrm{~s} 2 \mathrm{p}{ }^{3} \mathrm{P}-2 \mathrm{p}^{2}{ }^{3} \mathrm{P}$ multiplet at $1175 \AA$. However, this diagnostic is only sensitive up to log $N_{\mathrm{e}}=10\left(\mathrm{~cm}^{-3}\right)$ and is therefore not useful for active stars, that normally have higher densities. Moreover, temperature and opacity effects can be large. The C III 977/1176 ratio as measured from FUSE spectra is 1.0, much lower than the high-density limit (1.7), according to the C III CHIANTI model and calculated at $T=6.3 \times 10^{4} \mathrm{~K}$. This discrepancy can be interpreted in terms of opacity effects in the resonance line. The ratio is therefore not useful for density diagnostic for AU Mic, nor probably for diagnostic of other active stars. Young et al. (2001) and Ake et al. (2000) report FUSE measurements of Capella and $\mathrm{AB}$ Dor, a rapidly rotating active star. They report ratios of $2.0\left(N_{\mathrm{e}}=2 \times 10^{10} \mathrm{~cm}^{-3}\right)$ and 1.5, close to the highdensity limit, but also point out the uncertainties related to opacity effects. Schmitt et al. (1998) report ORFEUS observations of $\mathrm{AB}$ Dor, but measured a ratio in the range 1.0-1.3. The authors conclude that the AB Dor TR had densities in excess of $10^{11} \mathrm{~cm}^{-3}$. However, these ratio values are much lower than the high-density limit, and we believe that opacity effects are probably significant also in the ORFEUS spectrum, and no conclusions can be drawn.

\subsubsection{Summary of density diagnostics}

If we restrict to the FUV spectral range and consider the Capella and AU Mic observations as typical for active stars $\left(N_{\mathrm{e}}=5-10 \times 10^{10} \mathrm{~cm}^{-3}\right)$, we can conclude that: a) the best diagnostic is $\mathrm{O} V$, although with some uncertainties, due to the weakness of the $1371.292 \AA$ line and the temperature effects; b) results based on the O IV diagnostic should be treated with caution given the low density sensitivity and blending; c) the C III diagnostic is only useful at lower densities, and should be treated with caution because of opacity and temperature effects; d) the S IV diagnostic is only useful at higher densities; e) the Si III diagnostic has large temperature effects. Any measurement involving these ions should therefore be treated with caution and complemented with other methods. One possibility (see Sect. 5.3 below) is to use the EM loci method and the O III $1666.142 \AA$ and N IV $1486.496 \AA$ lines, that are density-sensitive (relative to dipole allowed transitions) in the $10^{10}-10^{11} \mathrm{~cm}^{-3}$ regime.

Another method, frequently adopted in the literature, is to use ratios of lines emitted by different elements. This leads to uncertain results unless independent methods for checking the ionisation state and the relative elemental abundances can be used. Temperature effects can also be important. For example, Doschek et al. (1978) and following authors (e.g. Cook \& Nicholas 1979) suggest the use of the Si III $1892 \AA / C$ III $1908 \AA$ ratio. As already mentioned, both C III and Si III lines have large temperature effects and therefore can only be used not only when the relative $\mathrm{C} / \mathrm{Si}$ abundance is known, but also when the $D E M$ is independently estimated. Cook \& Nicholas (1979) and following authors (e.g. Byrne et al. 1987) also suggest the use of the Si IV $1402 \AA / C$ III $1908 \AA$ ratio. In this case, aside from the same problems that the Si III/C III ratio has, this ratio is unreliable because the Si IV lines belong to the anomalous class.

\subsection{The DEM of $A U$ Mic in quiescence}

We have used the observations detailed in Sect. 4 to derive a $D E M$. As a first attempt, the photospheric abundances of Grevesse \& Sauval (1998) have been used, with a 


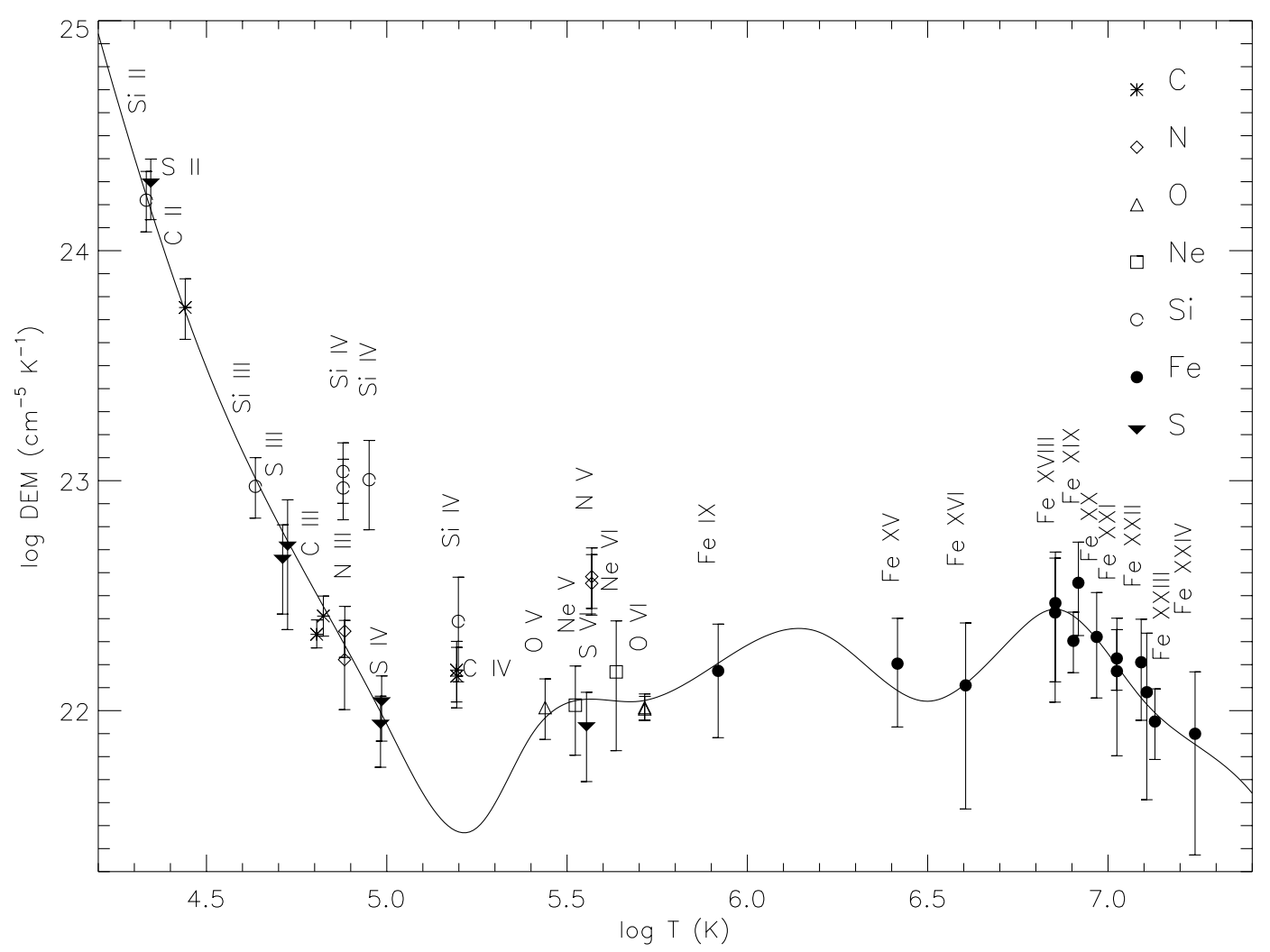

Fig. 7. The $D E M$ of AU Mic in quiescence, as derived from FUSE, STIS and EUVE spectra. Note the large deviations of the lines of the Li-like (C IV, N V) and Na-like (Si IV) ions.

correction for the oxygen abundance to a value of 8.73 (in the usual dex notation, with $\mathrm{H}$ fixed at 12 ), as recently revised by Grevesse (2002). Note that this value is much lower than the value of 8.93 published in Grevesse \& Anders (1991), which has been used as a reference in many previous studies. The contribution functions have been calculated at a constant electron pressure $P_{\mathrm{e}}=$ $1 \times 10^{16} \mathrm{~cm}^{-3} \mathrm{~K}$, for reasons that are explained below. The resulting $D E M$ is plotted in Fig. 7, while Table 3 presents the line list with the details and line identifications. Each experimental data point is over-plotted in Fig. 7 at the effective temperature $T_{\text {eff }}$ and at a value equal to the product $D E M\left(T_{\mathrm{eff}}\right) \times\left(I_{\mathrm{ob}} / I_{\mathrm{th}}\right)$. The error bars refer to the combination of observational and theoretical $(10 \%)$ errors and give an idea of the uncertainties in the derived $D E M$ values.

First, let us examine the $D E M$ at lower temperatures. The $D E M$ is well constrained in the $\log T=4-6$ range by all the lines observed by STIS and FUSE. The general agreement between the two sets of lines is very good, considering the lack of simultaneity. The DEM at temperatures as high as $10^{6} \mathrm{~K}$ is well constrained by the $\mathrm{O}$ VI lines, that have a long tail in their contribution function at high-temperatures. As in the case of the Sun, the $D E M$ has a minimum around $\log T=5.2$, and presents the usual increase toward million-degrees temperatures (see, e.g., Del Zanna \& Bromage 1999, for solar DEM distributions). The exact position of the minimum is not well constrained, since there are no observed allowed lines between the temperatures where S IV and O V are emitted. However, further constraints are given by N IV] and O IV], as discussed below. The S III, N III, S IV lines observed by FUSE confirm the anomalous behaviour of the lines of the $\mathrm{Li}$ and $\mathrm{Na}$ isoelectronic sequences. This anomalous behaviour is quite striking. The Li-like N V and C IV lines are underestimated by factors larger than 3 and 5 , respectively. The Na-like Si IV lines are also underestimated by a factor larger than 5 . The S VI $933.3 \AA$ (Na-like) is overestimated (only by a 40\%), while the O VI lines are the only ones that do not seem to present anomalous behaviour. This confirms the problems with these ions already found in the solar spectra.

In order to estimate the importance of density effects in the ion balance calculations for this case, we have performed a $D E M$ analysis using ionisation fractions calculated at a density $N_{\mathrm{e}}=10^{10} \mathrm{~cm}^{-3}$ (J. Raymond 2001, priv. comm.). As already shown by Vernazza \& Raymond (1978), density effects can be quite large. Indeed, we have found significant changes, not only to the calculated fluxes, but also to the effective temperatures. The fluxes of the Li- and Na-like lines increase (e.g. by factors of 2 for C IV and 3 for Si IV), suggesting that these density effects are in fact very important, although still not sufficient to completely remove the discrepancy in this particular case.

Our DEM distribution is in stark contrast with those obtained by Quin et al. (1993) and Pagano et al. (2000). 
The latter found a minimum in the $D E M$ at $\log T=4.7$, with a nearly flat $D E M$ until $\log T=5.4$, and concluded that the energy balance in the transition region of AU Mic is very different from that of the Sun. The large discrepancy in the results obtained by Pagano et al. (2000) is due to the fact that they used the Si III, Si IV, C IV, N V lines observed by STIS to constrain the log $T=4.5-5.5$ region. Pagano et al. presented the results in terms of the $I_{\mathrm{ob}} /\left(A_{\mathrm{b}} \times C\left(N_{\mathrm{e}}, T\right)\right)$ curves. For comparison, we show in Fig. 8 (top) these curves, together with the $E M_{\Delta \log T=0.3}$ values, i.e. the emission measures calculated with a $\Delta \log T=0.3$, for all the allowed lines, excluding those of the $\mathrm{Li}$ and $\mathrm{Na}$ isoelectronic sequences, that are plotted in Fig. 8 (middle). The erroneous $D E M$ derived by Pagano et al. (2000) resulted in the particularly high densities required to explain the fluxes of the density-sensitive lines of O III, N IV, O IV, O V $\left(\log N_{\mathrm{e}}=11.5-12\right)$ by using the emission measure loci. Figure 8 (bottom) clearly shows that the much lower densities found here are perfectly consistent with the emission measure values. We note that the use of the emission measure loci method can provide misleading results, since the $I_{\mathrm{ob}} /\left(A_{\mathrm{b}} \times C(T)\right)$ curves only provide upper limits to the emission measure. Instead it is important to calculate the line fluxes, i.e. the emissivities integrated over the $D E M$ distribution. In this respect, it is interesting to examine the behaviour of the line fluxes when the emissivities are calculated at different densities (or pressures). Figure 9 shows the results obtained when the emissivities are calculated at two constant densities $\left(N_{\mathrm{e}}=3.5\right.$ and $\left.14 \times 10^{10} \mathrm{~cm}^{-3}\right)$ and at constant pressure $\left(P_{\mathrm{e}}=10^{16} \mathrm{~cm}^{-3} \mathrm{~K}\right)$. Figure 9 shows that:

a) the $\mathrm{O}$ III] and $\mathrm{N} \mathrm{IV]} \mathrm{lines} \mathrm{are} \mathrm{very} \mathrm{sensitive} \mathrm{to} \mathrm{small}$ density variations;

b) the Si III] lines are very sensitive to both density and temperature effects;

c) it is impossible to reproduce all the intensities of the density sensitive lines if the emissivities are calculated at constant density;

d) a constant pressure $P_{\mathrm{e}}=10^{16} \mathrm{~cm}^{-3} \mathrm{~K}$ produces good agreement for all the transition region lines in the $\log T=$ 4.5-5.5 range (Si III], O III], N IV], O IV], O V], with the exception of S IV] 1406.016 $\AA$, that probably has an erroneous observed flux, see Sect. 4.2.1) and confirms the densities derived from the $L$-function method;

e) with this constant pressure, the N IV and O IV lines confirm the presence of a minimum at $\log T=5.2$ in the $D E M$.

At higher temperatures, the $D E M$ is not well constrained between $\log T=6-6.7$. The EUVE measurements suggest a peak at $\log T \simeq 6.1$ and a decrease at $\log T=6.5$. It is interesting to note that the Chandra and XMM gratings observe only few lines that cover this important temperature range, where the bulk of the quiet Sun emission is. The $D E M$ has a peak at $\log T=6.9$, well constrained by the FUSE and STIS data, together with the EUVE ones. This peak is typical of the coronae of active stars and of solar flares.
Regarding elemental abundances, no significant departures from the adopted solar photospheric abundances are found. This includes low-FIP elements ( $\mathrm{Si}$ ), medium-FIP elements $(\mathrm{S})$, and high-FIP ones $(\mathrm{C}, \mathrm{N}, \mathrm{O}, \mathrm{Ne})$. This is in stark contrast to the result obtained by Quin et al. (1993), where solar coronal abundances were found to be satisfactory. Again, this erroneous result was due to the use of the Si IV, C IV, N V lines in the DEM analysis.

Finally, it should be noted that erroneous $D E M$ distributions and chemical abundances such as those found for the AU Mic transition region by Quin et al. (1993) and Pagano et al. (2000) lead to inaccurate calculations of the radiative losses, and to misconceptions about physical processes that are derived from them.

\section{Conclusions}

We have discussed diagnostic methods and atomic data for the determination of densities, the DEM and the chemical composition of the transition regions and coronae of the Sun and active stars. We have described the severe limitations which some of the commonly used methods have. We have illustrated several issues with examples, mainly focusing on the diagnostic methods for the transition region using FUV (900-1700 $\AA$ - HST/STIS, FUSE) observations of AU Mic. We have complemented them with FUV observations of the Sun and Capella. We have revised some previous analyses and obtained results that are significantly different from those of other authors.

The problems highlighted are quite general and apply to other stellar transition regions (including the Sun) and to other wavelength ranges. In terms of emission measure analysis, we have indicated what we think is the best approach, which avoids frequently used approximations. We have described the main problems related to the direct determination of the relative elemental abundances, giving warnings about the use of emission measures and line ratio methods.

Regarding electron densities, we have discussed the limitations of using single line ratios, and recommended the use of the $L$-function method, which gives a better overall view of the results. We have presented the limitations in the use of lines that have a small density sensitivity, providing a discussion of the O IV lines at $1400 \AA$ as an example. The use of these lines is further complicated by the presence of an unknown blend.

We have shown the importance of revising earlier results by using more up-to-date atomic calculations. As an example, we have shown that the latest S IV calculations of Tayal (2000) provide density estimates that are significantly different from those reported in the previous literature.

The anomalous behaviour of lines from the $\mathrm{Li}$ and $\mathrm{Na}$ isoelectronic sequences has been neglected too often in previous solar and stellar publications. We have shown that the use of these lines produces erroneous results in the determination of: a) the $D E M$; b) the elemental abundances; c) the densities using the emission measure loci 

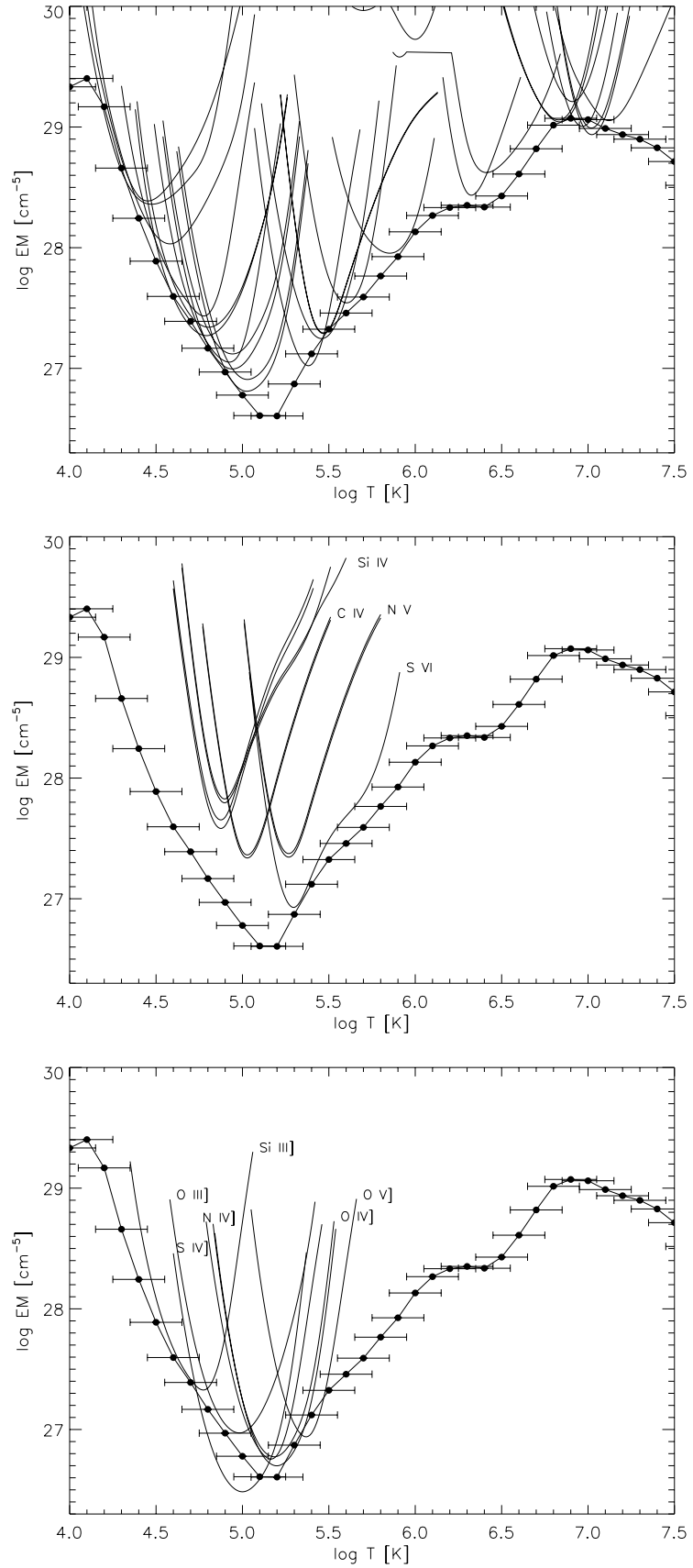

Fig. 8. The emission measures $E M_{\Delta \log T=0.3}$ values, together with the $I_{\mathrm{ob}} /\left(A_{\mathrm{b}} \times C(T)\right)$ curves, calculated at constant pressure $P_{\mathrm{e}}=10^{16} \mathrm{~cm}^{-3} \mathrm{~K}$. From top to bottom: the curves for the allowed lines; the curves for the anomalous allowed lines of the Li- and Na-like sequences; the curves for the forbidden lines.

method. Note that in most papers these lines have been the primary means of determining the physical characteristics of the solar and stellar transition regions.

In light of the above, we believe that a large body of work in terms of spectroscopic diagnostics of the solar and stellar outer atmospheres (and their application to
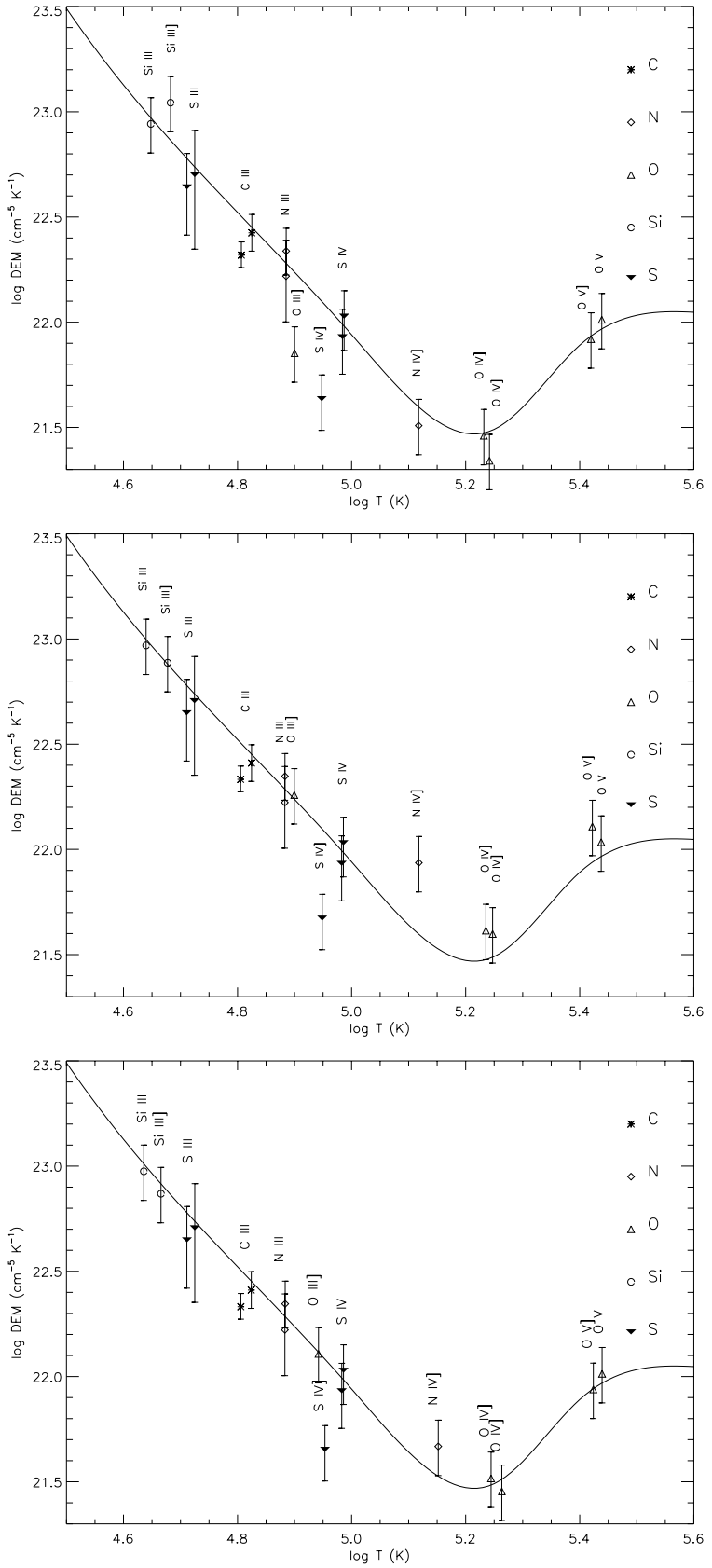

Fig. 9. The $D E M$ of the AU Mic in quiescence (same as Fig. 7), but with the emissivities calculated, from top to bottom: at constant density $N_{\mathrm{e}}=3.5 \times 10^{10} \mathrm{~cm}^{-3} ; N_{\mathrm{e}}=$ $14 \times 10^{10} \mathrm{~cm}^{-3}$; at constant pressure $P_{\mathrm{e}}=10^{16} \mathrm{~cm}^{-3} \mathrm{~K}$. Note the large variations in the forbidden lines Si III], O III], $\mathrm{N}$ IV], O IV], O V], and the fact that a constant pressure $P_{\mathrm{e}}=10^{16} \mathrm{~cm}^{-3} \mathrm{~K}$ produces the best agreement in the temperature range shown.

physical modeling) should be revisited, now that more accurate atomic and observational data are available.

Acknowledgements. G. Del Zanna and H. E. Mason acknowledge support from PPARC (UK). M. Landini and G. Del Zanna acknowledge support from ASI and MIUR (Italy). We thank R. Mewe for his helpful suggestions. 
Table 3. Results of the DEM analysis. The columns consist of: (1) the ion; (2) the theoretical wavelength $\lambda_{\text {th }}$; (3) the observed wavelength $\lambda_{\mathrm{ob}}$; (4) configuration and term description; (5) observed flux $I_{\mathrm{ob}}\left(10^{-14} \mathrm{ergs}_{\mathrm{cm}}^{-2} \mathrm{~s}^{-1}\right)$; (6) ratio between the theoretical $I_{\mathrm{th}}$ and the observed flux; (7) the estimated error on the ratio value; (8) the effective temperature (log values); (9) $T_{\max }$ (log values); (10) the contribution of each transition to the total calculated intensity of the line. (11) the instrument. The last column indicates: $(*)$ a selection of the observed allowed lines; (a) the anomalous allowed lines of the Li- and Na-like sequences; (d) the forbidden lines. The lines marked in the last column are plotted in Fig. 8.

\begin{tabular}{|c|c|c|c|c|c|c|c|c|c|c|c|}
\hline Ion & $\begin{array}{l}\lambda_{\mathrm{th}} \\
(\AA) \\
\end{array}$ & $\begin{array}{l}\lambda_{\mathrm{ob}} \\
(\AA) \\
\end{array}$ & Transition & $\begin{array}{c}I_{\mathrm{ob}} \\
\left(10^{-14}\right) \\
\end{array}$ & $\begin{array}{c}I_{\mathrm{th}} / \\
I_{\mathrm{ob}} \\
\end{array}$ & $+1-$ & $\begin{array}{l}\log \\
T_{\text {eff }} \\
\end{array}$ & $\begin{array}{l}\log \\
T_{\max } \\
\end{array}$ & frac & & \\
\hline Si II & 1533.430 & 1533.406 & $3 \mathrm{~s}^{2}\left({ }^{1} \mathrm{~S}\right) 3 \mathrm{p}^{2} \mathrm{P}_{3 / 2}-3 \mathrm{~s}^{2}\left({ }^{1} \mathrm{~S}\right) 4 \mathrm{~s}^{2} \mathrm{~S}_{1 / 2}$ & 0.69 & 1.09 & 0.24 & 4.28 & 4.43 & & STIS & \\
\hline Si II & 1526.706 & 1526.687 & $3 \mathrm{~s}^{2}\left({ }^{1} \mathrm{~S}\right) 3 \mathrm{p}^{2} \mathrm{P}_{1 / 2}-3 \mathrm{~s}^{2}\left({ }^{1} \mathrm{~S}\right) 4 \mathrm{~s}^{2} \mathrm{~S}_{1 / 2}$ & 0.58 & 0.66 & 0.15 & 4.28 & 4.43 & & STIS & \\
\hline Si II & 1304.369 & 1304.355 & $3 \mathrm{~s}^{2}\left({ }^{1} \mathrm{~S}\right) 3 \mathrm{p}{ }^{2} \mathrm{P}_{1 / 2}-3 \mathrm{~s} 3 \mathrm{p}^{2}{ }^{2} \mathrm{~S}_{1 / 2}$ & 0.12 & 1.05 & 0.23 & 4.33 & 4.48 & & STIS & $*$ \\
\hline Si II & 1309.274 & 1309.287 & $3 \mathrm{~s}^{2}\left({ }^{1} \mathrm{~S}\right) 3 \mathrm{p}{ }^{2} \mathrm{P}_{3 / 2}-3 \mathrm{~s} 3 \mathrm{p}^{2}{ }^{2} \mathrm{~S}_{1 / 2}$ & 0.32 & 0.78 & 0.17 & 4.33 & 4.48 & & STIS & \\
\hline Si II & 1264.737 & 1264.725 & $3 \mathrm{~s}^{2}\left({ }^{1} \mathrm{~S}\right) 3 \mathrm{p}{ }^{2} \mathrm{P}_{3 / 2}-3 \mathrm{~s}^{2}\left({ }^{1} \mathrm{~S}\right) 3 \mathrm{~d}^{2} \mathrm{D}_{5 / 2}$ & 0.48 & 2.01 & 0.45 & 4.35 & 4.49 & & STIS & \\
\hline Si II & 1265.001 & 1264.986 & $3 \mathrm{~s}^{2}\left({ }^{1} \mathrm{~S}\right) 3 \mathrm{p}^{2} \mathrm{P}_{3 / 2}-3 \mathrm{~s}^{2}\left({ }^{1} \mathrm{~S}\right) 3 \mathrm{~d}^{2} \mathrm{D}_{3 / 2}$ & 0.21 & 0.55 & 0.12 & 4.35 & 4.49 & & STIS & \\
\hline S II & 1253.811 & 1253.808 & $3 s^{2} 3 p^{3}{ }^{4} S_{3 / 2}-3 s 3 p^{4}{ }^{4} P_{3 / 2}$ & 0.12 & 1.65 & 0.37 & 4.34 & 4.45 & & STIS & \\
\hline S II & 1259.519 & 1259.495 & $3 s^{2} 3 p^{3}{ }^{4} \mathrm{~S}_{3 / 2}-3 \mathrm{~s} 3 \mathrm{p}^{4}{ }^{4} \mathrm{P}_{5 / 2}$ & 0.14 & 0.80 & 0.18 & 4.35 & 4.45 & & STIS & $*$ \\
\hline C II & 1334.524 & 1334.535 & $2 \mathrm{~s}^{2}\left({ }^{1} \mathrm{~S}\right) 2 \mathrm{p}^{2} \mathrm{P}_{1 / 2}-2 \mathrm{~s} 2 \mathrm{p}^{2}{ }^{2} \mathrm{D}_{3 / 2}$ & 6.89 & 1.00 & 0.22 & 4.44 & 4.58 & & STIS & \\
\hline C II & 1335.709 & 1335.692 & $2 \mathrm{~s}^{2}\left({ }^{1} \mathrm{~s}\right) 2 \mathrm{p}^{2} \mathrm{P}_{3 / 2}-2 \mathrm{~s} 2 \mathrm{p}^{2}{ }^{2} \mathrm{D}_{5 / 2}$ & 14.39 & 0.96 & 0.21 & 4.44 & 4.58 & & STIS & $*$ \\
\hline C II & 1036.332 & 1036.302 & $2 \mathrm{~s}^{2}\left({ }^{1} \mathrm{~S}\right) 2 \mathrm{p}{ }^{2} \mathrm{P}_{1 / 2}-2 \mathrm{~s} 2 \mathrm{p}^{2}{ }^{2} \mathrm{~S}_{1 / 2}$ & 0.73 & 1.06 & 0.17 & 4.53 & 4.63 & & FUSE & \\
\hline C II & 1037.020 & 1036.982 & $2 \mathrm{~s}^{2}\left({ }^{1} \mathrm{~S}\right) 2 \mathrm{p}^{2} \mathrm{P}_{3 / 2}-2 \mathrm{~s} 2 \mathrm{p}^{2}{ }^{2} \mathrm{~S}_{1 / 2}$ & 1.1 & 1.39 & 0.18 & 4.53 & 4.63 & & FUSE & \\
\hline C II & 1323.952 & 1323.929 & $2 \mathrm{~s} 2 \mathrm{p}^{2}{ }^{2} \mathrm{D}_{5 / 2}-2 \mathrm{p}^{3}{ }^{2} \mathrm{D}_{5 / 2}$ & 0.14 & 0.95 & 0.21 & 4.63 & 4.69 & 0.56 & STIS & \\
\hline C II & 1323.907 & & $2 \mathrm{~s} 2 \mathrm{p}^{2}{ }^{2} \mathrm{D}_{3 / 2}-2 \mathrm{p}^{3}{ }^{2} \mathrm{D}_{3 / 2}$ & & & & & 4.69 & 0.36 & & \\
\hline Si III & 1206.499 & 1206.496 & $3 \mathrm{~s}^{2}{ }^{1} \mathrm{~S}_{0}-3 \mathrm{~s} 3 \mathrm{p}{ }^{1} \mathrm{P}_{1}$ & 7.9 & 1.09 & 0.24 & 4.64 & 4.78 & & STIS & $*$ \\
\hline Si III & 1301.147 & 1301.139 & $3 \mathrm{~s} 3 \mathrm{p}^{3} \mathrm{P}_{1}-3 \mathrm{p}^{2}{ }^{3} \mathrm{P}_{0}$ & 0.17 & 0.65 & 0.14 & 4.66 & 4.77 & & STIS & \\
\hline Si III & 1303.323 & 1303.325 & $3 \mathrm{~s} 3 \mathrm{p}^{3} \mathrm{P}_{2}-3 \mathrm{p}^{2}{ }^{3} \mathrm{P}_{1}$ & 0.15 & 1.05 & 0.23 & 4.66 & 4.77 & & STIS & \\
\hline Si III & 1298.944 & 1298.938 & $3 \mathrm{~s} 3 \mathrm{p}^{3} \mathrm{P}_{2}-3 \mathrm{p}^{2}{ }^{3} \mathrm{P}_{2}$ & 0.51 & 1.11 & 0.25 & 4.67 & 4.77 & 0.83 & STIS & $\mathrm{d}$ \\
\hline Si III & 1298.892 & & $3 \mathrm{~s} 3 \mathrm{p}^{3} \mathrm{P}_{1}-3 \mathrm{p}^{2}{ }^{3} \mathrm{P}_{1}$ & & & & & 4.77 & 0.17 & & \\
\hline Si III & 1294.543 & 1294.537 & $3 \mathrm{~s} 3 \mathrm{p}^{3} \mathrm{P}_{1}-3 \mathrm{p}^{2}{ }^{3} \mathrm{P}_{2}$ & 0.21 & 0.76 & 0.17 & 4.67 & 4.77 & & STIS & \\
\hline Si III & 1296.726 & 1296.713 & $3 \mathrm{~s} 3 \mathrm{p}^{3} \mathrm{P}_{0}-3 \mathrm{p}^{2}{ }^{3} \mathrm{P}_{1}$ & 0.08 & 1.60 & 0.36 & 4.67 & 4.77 & & STIS & \\
\hline Si III & 1109.969 & 1110.006 & $3 \mathrm{~s} 3 \mathrm{p}^{3} \mathrm{P}_{1}-3 \mathrm{~s} 3 \mathrm{~d}^{3} \mathrm{D}_{2}$ & 0.25 & 1.27 & 0.28 & 4.69 & 4.78 & 0.74 & FUSE & \\
\hline Si III & 1109.939 & & $3 \mathrm{~s} 3 \mathrm{p}^{3} \mathrm{P}_{1}-3 \mathrm{~s} 3 \mathrm{~d}^{3} \mathrm{D}_{1}$ & & & & & 4.78 & 0.26 & & \\
\hline Si III & 1108.357 & 1108.370 & $3 \mathrm{~s} 3 \mathrm{p}^{3} \mathrm{P}_{0}-3 \mathrm{~s} 3 \mathrm{~d}^{3} \mathrm{D}_{1}$ & 0.083 & 1.32 & 0.68 & 4.69 & 4.78 & & FUSE & \\
\hline Si III & 1113.229 & 1113.270 & $3 \mathrm{~s} 3 \mathrm{p}^{3} \mathrm{P}_{2}-3 \mathrm{~s} 3 \mathrm{~d}^{3} \mathrm{D}_{3}$ & 0.31 & 2.00 & 0.39 & 4.69 & 4.78 & 0.87 & FUSE & \\
\hline Si III & 1113.203 & & $3 \mathrm{~s} 3 \mathrm{p}^{3} \mathrm{P}_{2}-3 \mathrm{~s} 3 \mathrm{~d}^{3} \mathrm{D}_{2}$ & & & & & 4.78 & 0.13 & & \\
\hline S III & 1021.321 & 1021.357 & $3 \mathrm{~s}^{2} 3 \mathrm{p}^{2}{ }^{3} \mathrm{P}_{2}-3 \mathrm{~s} 3 \mathrm{p}^{3}{ }^{3} \mathrm{P}_{2}$ & 0.083 & 1.38 & 0.48 & 4.71 & 4.79 & & FUSE & $*$ \\
\hline S III & 1077.171 & 1077.017 & $3 s^{2} 3 p^{2}{ }^{1} D_{2}-3 s^{2} 3 p 3 d^{1} D_{2}$ & 0.13 & 1.10 & 0.56 & 4.72 & 4.80 & & FUSE & $*$ \\
\hline C III & 977.022 & 977.015 & $2 \mathrm{~s}^{2}{ }^{1} \mathrm{~S}_{0}-2 \mathrm{~s} 2 \mathrm{p}{ }^{1} \mathrm{P}_{1}$ & 12 & 1.49 & 0.16 & 4.81 & 4.92 & & FUSE & \\
\hline C III & 1174.935 & 1174.939 & $2 \mathrm{~s} 2 \mathrm{p}^{3} \mathrm{P}_{1}-2 \mathrm{p}^{2}{ }^{3} \mathrm{P}_{2}$ & 2 & 0.70 & 0.10 & 4.82 & 4.92 & & FUSE & \\
\hline C III & 1175.265 & 1175.281 & $2 \mathrm{~s} 2 \mathrm{p}{ }^{3} \mathrm{P}_{0}-2 \mathrm{p}^{2}{ }^{3} \mathrm{P}_{1}$ & 1.6 & 0.70 & 0.10 & 4.82 & 4.92 & & FUSE & \\
\hline C III & 1175.592 & 1175.570 & $2 \mathrm{~s} 2 \mathrm{p}^{3} \mathrm{P}_{1}-2 \mathrm{p}^{2}{ }^{3} \mathrm{P}_{1}$ & 1.1 & 0.76 & 0.11 & 4.82 & 4.92 & & FUSE & \\
\hline C III & 1175.713 & 1175.709 & $2 \mathrm{~s} 2 \mathrm{p}^{3} \mathrm{P}_{2}-2 \mathrm{p}^{2}{ }^{3} \mathrm{P}_{2}$ & 3.8 & 1.10 & 0.16 & 4.82 & 4.92 & & FUSE & $*$ \\
\hline C III & 1175.989 & 1175.986 & $2 \mathrm{~s} 2 \mathrm{p}^{3} \mathrm{P}_{1}-2 \mathrm{p}^{2}{ }^{3} \mathrm{P}_{0}$ & 1.8 & 0.62 & 0.09 & 4.82 & 4.92 & & FUSE & \\
\hline C III & 1176.372 & 1176.366 & $2 \mathrm{~s} 2 \mathrm{p}^{3} \mathrm{P}_{2}-2 \mathrm{p}^{2}{ }^{3} \mathrm{P}_{1}$ & 1.6 & 0.87 & 0.12 & 4.82 & 4.92 & & FUSE & \\
\hline C III & 1175.713 & 1175.694 & 2s $2 \mathrm{p}^{3} \mathrm{P}_{2}-2 \mathrm{p}^{2}{ }^{3} \mathrm{P}_{2}$ & 3.98 & 1.05 & 0.24 & 4.82 & 4.92 & & STIS & \\
\hline Si IV & 1402.770 & 1402.747 & $3 \mathrm{~s}^{2} \mathrm{~S}_{1 / 2}-3 \mathrm{p}{ }^{2} \mathrm{P}_{1 / 2}$ & 2.92 & 0.18 & 0.04 & 4.88 & 4.87 & & STIS & $\mathrm{a}$ \\
\hline Si IV & 1393.755 & 1393.744 & $3 \mathrm{~s}^{2} \mathrm{~S}_{1 / 2}-3 \mathrm{p}{ }^{2} \mathrm{P}_{3 / 2}$ & 4.93 & 0.21 & 0.05 & 4.88 & 4.88 & & STIS & $\mathrm{a}$ \\
\hline Si IV & 1128.325 & 1128.350 & $3 \mathrm{p}^{2} \mathrm{P}_{3 / 2}-3 \mathrm{~d}^{2} \mathrm{D}_{5 / 2}$ & 0.2 & 0.12 & 0.04 & 4.95 & 4.90 & 0.90 & FUSE & $\mathrm{a}$ \\
\hline Si IV & 1128.340 & & $3 \mathrm{p}^{2} \mathrm{P}_{3 / 2}-3 \mathrm{~d}^{2} \mathrm{D}_{3 / 2}$ & & & & & 4.90 & 0.10 & & \\
\hline Si IV & 1122.500 & 1122.578 & $3 \mathrm{p}^{2} \mathrm{P}_{1 / 2}-3 \mathrm{~d}{ }^{2} \mathrm{D}_{3 / 2}$ & 0.11 & 0.12 & 0.05 & 5.20 & 4.90 & & FUSE & $\mathrm{a}$ \\
\hline N III & 989.787 & 989.752 & $2 \mathrm{~s}^{2} 2 \mathrm{p}^{2} \mathrm{P}_{1 / 2}-2 \mathrm{~s} 2 \mathrm{p}^{2}{ }^{2} \mathrm{D}_{3 / 2}$ & 0.46 & 1.16 & 0.40 & 4.88 & 4.94 & & FUSE & $*$ \\
\hline N III & 991.564 & 991.538 & $2 \mathrm{~s}^{2} 2 \mathrm{p}^{2} \mathrm{P}_{3 / 2}-2 \mathrm{~s} 2 \mathrm{p}^{2}{ }^{2} \mathrm{D}_{5 / 2}$ & 1.2 & 0.87 & 0.16 & 4.88 & 4.94 & 0.90 & FUSE & $*$ \\
\hline N III & 991.495 & & $2 \mathrm{~s}^{2} 2 \mathrm{p}^{2} \mathrm{P}_{3 / 2}-2 \mathrm{~s} 2 \mathrm{p}^{2}{ }^{2} \mathrm{D}_{3 / 2}$ & & & & & 4.94 & 0.10 & & \\
\hline O III & 1666.142 & 1666.109 & $2 \mathrm{~s}^{2} 2 \mathrm{p}^{2}{ }^{3} \mathrm{P}_{2}-2 \mathrm{~s} 2 \mathrm{p}^{3}{ }^{5} \mathrm{~S}_{2}$ & 0.13 & 1.02 & 0.23 & 4.94 & 4.98 & & STIS & $\mathrm{d}$ \\
\hline S IV & 1406.016 & 1406.031 & $3 \mathrm{~s}^{2} 3 \mathrm{p}^{2} \mathrm{P}_{3 / 2}-3 \mathrm{~s} 3 \mathrm{p}^{2}{ }^{4} \mathrm{P}_{5 / 2}$ & 0.04 & 2.76 & 0.62 & 4.95 & 5.00 & & STIS & $\mathrm{d}$ \\
\hline S IV & 1072.974 & 1072.930 & $3 \mathrm{~s}^{2} 3 \mathrm{p}^{2} \mathrm{P}_{3 / 2}-3 \mathrm{~s} 3 \mathrm{p}^{2}{ }^{2} \mathrm{D}_{5 / 2}$ & 0.23 & 1.18 & 0.32 & 4.98 & 5.03 & & FUSE & $*$ \\
\hline S IV & 1062.664 & 1062.572 & $3 \mathrm{~s}^{2} 3 \mathrm{p}{ }^{2} \mathrm{P}_{1 / 2}-3 \mathrm{~s} 3 \mathrm{p}^{2}{ }^{2} \mathrm{D}_{3 / 2}$ & 0.19 & 0.93 & 0.23 & 4.99 & 5.03 & & FUSE & $*$ \\
\hline N IV & 1486.496 & 1486.510 & $2 \mathrm{~s}^{2}{ }^{1} \mathrm{~S}_{0}-2 \mathrm{~s} 2 \mathrm{p}{ }^{3} \mathrm{P}_{1}$ & 0.09 & 0.72 & 0.16 & 5.15 & 5.16 & & STIS & $\mathrm{d}$ \\
\hline C IV & 1550.772 & 1550.746 & $1 s^{2} 2 s^{2} S_{1 / 2}-1 s^{2} 2 p^{2} P_{1 / 2}$ & 11.34 & 0.20 & 0.04 & 5.19 & 5.03 & & STIS & $\mathrm{a}$ \\
\hline C IV & 1548.201 & 1548.181 & $1 \mathrm{~s}^{2} 2 \mathrm{~s}^{2} \mathrm{~S}_{1 / 2}-1 \mathrm{~s}^{2} 2 \mathrm{p}^{2} \mathrm{P}_{3 / 2}$ & 21.36 & 0.21 & 0.05 & 5.19 & 5.03 & & STIS & $\mathrm{a}$ \\
\hline O IV & 1404.793 & 1404.811 & $2 \mathrm{~s}^{2} 2 \mathrm{p}^{2} \mathrm{P}_{3 / 2}-2 \mathrm{~s} 2 \mathrm{p}^{2}{ }^{4} \mathrm{P}_{3 / 2}$ & 0.11 & 1.15 & 0.26 & 5.22 & 5.21 & 0.79 & STIS & \\
\hline S IV & 1404.808 & & $3 s^{2} 3 p{ }^{2} \mathrm{P}_{1 / 2}-3 \mathrm{~s} 3 \mathrm{p}^{2}{ }^{4} \mathrm{P}_{1 / 2}$ & & & & & 4.99 & 0.21 & & \\
\hline O IV & 1399.779 & 1399.765 & $2 \mathrm{~s}^{2} 2 \mathrm{p}^{2} \mathrm{P}_{1 / 2}-2 \mathrm{~s} 2 \mathrm{p}^{2}{ }^{4} \mathrm{P}_{1 / 2}$ & 0.07 & 1.72 & 0.39 & 5.24 & 5.18 & & STIS & \\
\hline O IV & 1407.383 & 1407.361 & $2 \mathrm{~s}^{2} 2 \mathrm{p}^{2} \mathrm{P}_{3 / 2}-2 \mathrm{~s} 2 \mathrm{p}^{2}{ }^{4} \mathrm{P}_{1 / 2}$ & 0.12 & 0.93 & 0.21 & 5.24 & 5.18 & & STIS & $\mathrm{d}$ \\
\hline O IV & 1401.171 & 1401.140 & $2 \mathrm{~s}^{2} 2 \mathrm{p}^{2} \mathrm{P}_{3 / 2}-2 \mathrm{~s} 2 \mathrm{p}^{2}{ }^{4} \mathrm{P}_{5 / 2}$ & 0.4 & 1.14 & 0.25 & 5.26 & 5.20 & & STIS & $\mathrm{d}$ \\
\hline $\mathrm{OV}$ & 1218.390 & 1218.322 & $2 \mathrm{~s}^{2}{ }^{1} \mathrm{~S}_{0}-2 \mathrm{~s} 2 \mathrm{p}{ }^{3} \mathrm{P}_{1}$ & 1.83 & 1.01 & 0.23 & 5.42 & 5.37 & & STIS & $\mathrm{d}$ \\
\hline $\mathrm{OV}$ & 1371.292 & 1371.285 & $2 \mathrm{~s} 2 \mathrm{p}{ }^{1} \mathrm{P}_{1}-2 \mathrm{p}^{2}{ }^{1} \mathrm{D}_{2}$ & 0.35 & 0.90 & 0.20 & 5.44 & 5.38 & & STIS & $*$ \\
\hline
\end{tabular}


Table 3. continued.

\begin{tabular}{|c|c|c|c|c|c|c|c|c|c|c|c|}
\hline Ion & $\begin{array}{l}\lambda_{\text {th }} \\
(\AA)\end{array}$ & $\begin{array}{l}\lambda_{\mathrm{ob}} \\
(\AA)\end{array}$ & Transition & $\begin{array}{c}I_{\mathrm{ob}} \\
\left(10^{-14}\right)\end{array}$ & $\begin{array}{r}I_{\mathrm{th}} / \\
I_{\mathrm{ob}} \\
\end{array}$ & $+1-$ & $\begin{array}{l}\log \\
T_{\text {eff }} \\
\end{array}$ & $\begin{array}{l}\log \\
T_{\max }\end{array}$ & frac & & \\
\hline $\mathrm{Ne} \mathrm{V}$ & 1145.596 & 1145.624 & $2 \mathrm{~s}^{2} 2 \mathrm{p}^{2}{ }^{3} \mathrm{P}_{2}-2 \mathrm{~s} 2 \mathrm{p}^{3}{ }^{5} \mathrm{~S}_{2}$ & 0.15 & 1.05 & 0.36 & 5.52 & 5.46 & & FUSE & $*$ \\
\hline S VI & 933.378 & 933.397 & $3 \mathrm{~s}^{2} \mathrm{~S}_{1 / 2}-3 \mathrm{p}{ }^{2} \mathrm{P}_{3 / 2}$ & 0.48 & 1.38 & 0.48 & 5.55 & 5.30 & & FUSE & $\mathrm{a}$ \\
\hline $\mathrm{N}$ V & 1242.804 & 1242.794 & $1 \mathrm{~s}^{2} 2 \mathrm{~s}^{2} \mathrm{~S}_{1 / 2}-1 \mathrm{~s}^{2} 2 \mathrm{p}^{2} \mathrm{P}_{1 / 2}$ & 2.11 & 0.31 & 0.07 & 5.57 & 5.27 & & STIS & $\mathrm{a}$ \\
\hline $\mathrm{N}$ V & 1238.821 & 1238.799 & $1 \mathrm{~s}^{2} 2 \mathrm{~s}^{2} \mathrm{~S}_{1 / 2}-1 \mathrm{~s}^{2} 2 \mathrm{p}^{2} \mathrm{P}_{3 / 2}$ & 4.52 & 0.29 & 0.07 & 5.57 & 5.27 & & STIS & $\mathrm{a}$ \\
\hline $\mathrm{Ne}$ VI & 999.182 & 999.181 & $2 \mathrm{~s}^{2} 2 \mathrm{p}^{2} \mathrm{P}_{3 / 2}-2 \mathrm{~s} 2 \mathrm{p}^{2}{ }^{4} \mathrm{P}_{5 / 2}$ & 0.45 & 0.75 & 0.38 & 5.64 & 5.60 & & FUSE & $*$ \\
\hline $\mathrm{O}$ VI & 1037.615 & 1037.583 & $1 \mathrm{~s}^{2} 2 \mathrm{~s}^{2} \mathrm{~S}_{1 / 2}-1 \mathrm{~s}^{2} 2 \mathrm{p}^{2} \mathrm{P}_{1 / 2}$ & 9.6 & 1.07 & 0.11 & 5.72 & 5.47 & & FUSE & * \\
\hline $\mathrm{O} \mathrm{VI}$ & 1031.914 & 1031.920 & $1 \mathrm{~s}^{2} 2 \mathrm{~s}^{2} \mathrm{~S}_{1 / 2}-1 \mathrm{~s}^{2} 2 \mathrm{p}^{2} \mathrm{P}_{3 / 2}$ & 19 & 1.09 & 0.11 & 5.72 & 5.47 & & FUSE & $*$ \\
\hline Fe IX & 171.073 & 171.000 & $3 \mathrm{p} 6{ }^{1} \mathrm{~S}_{0}-3 \mathrm{p} 5.3 \mathrm{~d}{ }^{1} \mathrm{P}_{1}$ & 22.9 & 1.08 & 0.48 & 5.92 & 5.85 & & EUVE & * \\
\hline Fe XV & 284.160 & 284.200 & $3 \mathrm{~s} 2{ }^{1} \mathrm{~S}_{0}-3 \mathrm{~s} 3 \mathrm{p}{ }^{1} \mathrm{P}_{1}$ & 40.8 & 0.76 & 0.33 & 6.42 & 6.32 & & EUVE & $*$ \\
\hline Fe XVI & 335.410 & 335.300 & $3 \mathrm{~s}^{2} \mathrm{~S}_{1 / 2}-3 \mathrm{p}{ }^{2} \mathrm{P}_{3 / 2}$ & 27.9 & 1.02 & 0.70 & 6.61 & 6.41 & & EUVE & $*$ \\
\hline Fe XVIII & 93.923 & 94.100 & $2 \mathrm{~s}^{2} 2 \mathrm{p}^{5}{ }^{2} \mathrm{P}_{3 / 2}-2 \mathrm{~s} 2 \mathrm{p}^{6}{ }^{2} \mathrm{~S}_{1 / 2}$ & 8.7 & 1.03 & 0.58 & 6.85 & 6.83 & & EUVE & * \\
\hline Fe XVIII & 974.860 & 974.810 & $2 \mathrm{~s}^{2} 2 \mathrm{p}^{5}{ }^{2} \mathrm{P}_{3 / 2}-2 \mathrm{~s}^{2} 2 \mathrm{p}^{5}{ }^{2} \mathrm{P}_{1 / 2}$ & 0.48 & 0.94 & 0.48 & 6.85 & 6.83 & & EUVE & $*$ \\
\hline Fe XIX & 1118.057 & 1118.013 & $2 \mathrm{~s}^{2} 2 \mathrm{p}^{4}{ }^{3} \mathrm{P}_{2}-2 \mathrm{~s}^{2} 2 \mathrm{p}^{4}{ }^{3} \mathrm{P}_{1}$ & 0.22 & 1.28 & 0.29 & 6.90 & 6.90 & & FUSE & * \\
\hline Fe XIX & 108.355 & 108.500 & $2 \mathrm{~s}^{2} 2 \mathrm{p}^{4}{ }^{3} \mathrm{P}_{2}-2 \mathrm{~s} 2 \mathrm{p}^{5}{ }^{3} \mathrm{P}_{2}$ & 7.96 & 0.69 & 0.25 & 6.92 & 6.90 & & EUVE & $*$ \\
\hline Fe XX & 121.845 & 121.700 & $2 \mathrm{~s}^{2} 2 \mathrm{p}^{3}{ }^{4} \mathrm{~S}_{3 / 2}-2 \mathrm{~s} 2 \mathrm{p}^{4}{ }^{4} \mathrm{P}_{3 / 2}$ & 6.2 & 0.98 & 0.41 & 6.97 & 6.97 & & EUVE & $*$ \\
\hline Fe XXI & 1354.080 & 1354.045 & $2 \mathrm{p}^{2}{ }^{3} \mathrm{P}_{0}-2 \mathrm{p}^{2}{ }^{3} \mathrm{P}_{1}$ & 0.77 & 0.93 & 0.21 & 7.02 & 7.02 & & STIS & $*$ \\
\hline Fe XXI & 128.752 & 128.600 & $2 \mathrm{p}^{2}{ }^{3} \mathrm{P}_{0}-2 \mathrm{~s} 2 \mathrm{p}^{3}{ }^{3} \mathrm{D}_{1}$ & 8.7 & 1.05 & 0.57 & 7.02 & 7.02 & & EUVE & * \\
\hline Fe XXII & 135.755 & 135.800 & $2 \mathrm{~s}^{2} 2 \mathrm{p}^{2} \mathrm{P}_{1 / 2}-1 \mathrm{~s}^{2} 2 \mathrm{~s} 2 \mathrm{p}^{2}{ }^{2} \mathrm{D}_{3 / 2}$ & 7.8 & 0.70 & 0.28 & 7.09 & 7.08 & & EUVE & $*$ \\
\hline Fe XXI & 117.499 & 117.600 & $2 \mathrm{p}^{2}{ }^{3} \mathrm{P}_{1}-2 \mathrm{~s} 2 \mathrm{p}^{3}{ }^{3} \mathrm{P}_{1}$ & 3.2 & 0.89 & 0.56 & 7.11 & 7.02 & 0.50 & EUVE & \\
\hline Ni XXV & 117.911 & & $2 \mathrm{~s}^{2}{ }^{1} \mathrm{~S}_{0}-2 \mathrm{~s} 2 \mathrm{p}{ }^{1} \mathrm{P}_{1}$ & & & & & 7.24 & 0.28 & & \\
\hline Ni XXII & 117.918 & & $2 s^{2} 2 p^{3}{ }^{4} S_{3 / 2}-2 s 2 p^{4}{ }^{4} S_{5 / 2}$ & & & & & 7.05 & 0.11 & & \\
\hline Fe XXIII & 132.906 & 132.800 & $2 \mathrm{~s}^{2}{ }^{1} \mathrm{~S}_{0}-2 \mathrm{~s} 2 \mathrm{p}^{1} \mathrm{P}_{1}$ & 22.6 & 1.09 & 0.29 & 7.13 & 7.14 & 0.67 & EUVE & $*$ \\
\hline Fe XX & 132.840 & & $2 \mathrm{~s}^{2} 2 \mathrm{p}^{3}{ }^{4} \mathrm{~S}_{3 / 2}-2 \mathrm{~s} 2 \mathrm{p}^{4}{ }^{4} \mathrm{P}_{5 / 2}$ & & & & & 6.97 & 0.31 & & \\
\hline Fe XXIV & 192.029 & 191.700 & $1 \mathrm{~s}^{2} 2 \mathrm{~s}^{2} \mathrm{~S}_{1 / 2}-1 \mathrm{~s}^{2} 2 \mathrm{p}^{2} \mathrm{P}_{3 / 2}$ & 10.4 & 0.90 & 0.61 & 7.24 & 7.23 & 0.84 & EUVE & \\
\hline S XI & 191.266 & & $2 \mathrm{~s} 22 \mathrm{p} 2{ }^{3} \mathrm{P}_{2}-2 \mathrm{~s} 2 \mathrm{p} 3{ }^{3} \mathrm{~S}_{1}$ & & & & & 6.28 & 0.14 & & \\
\hline
\end{tabular}

\section{References}

Ake, T. B., Dupree, A. K., Young, P. R., et al. 2000, ApJ, 538, L87

Arnaud, M., \& Raymond, J. 1992, ApJ, 398, 394

Arnaud, M., \& Rothenflug, R. 1985, A\&AS, 60, 425

Ayres, T. R., Brown, A., Osten, R. A., et al. 2001, ApJ, 549, 554

Ayres, T. R., Eriksson, K., Linsky, J. L., \& Stencel, R. E. 1983, ApJ, 270, L17

Berrington, K. A., Burke, P. G., Dufton, P. L., \& Kingston, A. E. 1985, Atomic Data and Nuclear Data Tables, 33, 195

Brage, T., Judge, P. G., \& Brekke, P. 1996, ApJ, 464, 1030

Brandt, J. C., Heap, S. R., Walter, F. M., et al. 2001, AJ, 121, 2173

Brooks, D. H., Fischbacher, G. A., Fludra, A., et al. 2000, A\&A, 357, 697

Brown, A., de M. Ferraz, M. C., \& Jordan, C. 1984a, MNRAS, 207, 831

Brown, A., Jordan, C., Stencel, R. E., Linsky, J. L., \& Ayres, T. R. 1984b, ApJ, 283, 731

Burgess, A., \& Summers, H. P. 1969, ApJ, 157, 1007

Burton, W. M., Jordan, C., Ridgeley, A., \& Wilson, R. 1971, Phil. Trans. Roy. Soc. Lon. A, 270, 81

Butler, C. J., Doyle, J. G., Andrews, A. D., et al. 1987, A\&A, 174, 139

Byrne, P. B., Doyle, J. G., Brown, A., Linsky, J. L., \& Rodono, M. 1987, A\&A, 180, 172

Cook, J. W., Cheng, C.-C., Jacobs, V. L., \& Antiochos, S. K. 1989, ApJ, 338, 1176

Cook, J. W., Keenan, F. P., Dufton, P. L., et al. 1995, ApJ, 444,936

Cook, J. W., \& Nicolas, K. R. 1979, ApJ, 229, 1163

Craig, I. J. D., \& Brown, J. C. 1986, Inverse problems in as- tronomy: A guide to inversion strategies for remotely sensed data (Research supported by SERC. Bristol, England and Boston, MA, Adam Hilger, Ltd., 1986, 159 p.)

Del Zanna, G. 1999, Ph.D. Thesis, Univ. of Central Lancashire, UK

Del Zanna, G., \& Bromage, B. J. I. 1999, J. Geophys. Res., 104, 9753

Del Zanna, G., Bromage, B. J. I., Landi, E., \& Landini, M. 2001a, A\&A, 379, 708

Del Zanna, G., Bromage, B. J. I., \& Mason, H. E. 2001b, in Solar and Galactic Composition, AIP Conf. Proc., 598, 59

Del Zanna, G., Landini, M., Migliorini, S., \& Monsignori Fossi, B. 1995, in PASP Conf. Ser., 109, 261

Dere, K. P., Landi, E., Mason, H. E., Monsignori Fossi, B. C., \& Young, P. R. 1997, A\&AS, 125, 149

Dere, K. P., Landi, E., Young, P. R., \& Del Zanna, G. 2001, ApJS, 134, 331

Doschek, E. E., Laming, J. M., Doschek, G. A., Feldman, U., \& Wilhelm, K. 1999, ApJ, 518, 909

Doschek, G. A. 1984, ApJ, 279, 446

Doschek, G. A. 1997, ApJ, 476, 903

Doschek, G. A., \& Feldman, U. 1977, A\&A, 58, L13

Doschek, G. A., Feldman, U., Mariska, J. T., \& Linsky, J. L. 1978, ApJ, 226, L35

Doyle, J. G., \& McWhirter, R. W. P. 1980, MNRAS, 193, 947

Doyle, J. G., \& Raymond, J. C. 1984, Sol. Phys., 90, 97

Drake, J. J., Laming, J. M., \& Widing, K. G. 1995, ApJ, 443, 393

Dufton, P. L., Hibbert, A., Kingston, A. E., \& Doschek, G. A. 1982, ApJ, 257, 338

Dupree, A. K. 1972, ApJ, 178, 527

Feldman, U. 1992, Phys. Scr., 46, 202

Feldman, U., \& Doschek, G. A. 1979, A\&A, 79, 357 
Feldman, U., Doschek, G. A., \& Rosenberg, F. D. 1977, ApJ, 215,652

Feldman, U., \& Laming, J. M. 2000, Phys. Scr., 61, 222

Flower, D. R., \& Nussbaumer, H. 1975, A\&A, 45, 145

Grevesse, N. 2002, Adv. Space Res., in press

Grevesse, N., \& Anders, E. 1991 Solar interior and atmosphere (Tucson, AZ, University of Arizona Press), 1227

Grevesse, N., \& Sauval, A. J. 1998, Space Sci. Rev., 85, 161

Griffiths, N. W., \& Jordan, C. 1998, ApJ, 497, 883

Harper, G. M., Jordan, C., Judge, P. G., et al. 1999, MNRAS, 303, L41

Harper, G. M., Wilkinson, E., Brown, A., Jordan, C., \& Linsky, J. L. 2001, ApJ, 551, 486

Hartmann, L., Dupree, A. K., Jordan, C., \& Brown, A. 1985, ApJ, 296, 576

Jordan, C. 1967, Sol. Phys., 2, 441

Jordan, C. 1985, MNRAS, 214, 1P

Jordan, C. 2000, Plasma Physics and Controlled Fusion, 42, 415

Jordan, C., Ayres, T. R., Brown, A., Linsky, J. L., \& Simon, T. 1987, MNRAS, 225, 903

Judge, P. G. 2000, ApJ, 531, 585

Judge, P. G., \& McIntosh, S. W. 1999, Sol. Phys., 190, 331

Judge, P. G., Woods, T. N., Brekke, P., \& Rottman, G. J. 1995, ApJ, 455, L85

Keenan, F. P., \& Kingston, A. E. 1986, MNRAS, 220, 493

Laming, J. M., \& Feldman, U. 1999, ApJ, 527, 461

Landi, E., \& Landini, M. 1998, A\&A, 340, 265

Linsky, J. L., Bornmann, P. L., Carpenter, K. G., et al. 1982, ApJ, 260, 670

Linsky, J. L., Neff, J. E., Brown, A., et al. 1989, A\&A, 211, 173

Linsky, J. L., Wood, B. E., Judge, P., et al. 1995, ApJ, 442, 381

Maran, S. P., Robinson, R. D., Shore, S. N., et al. 1994, ApJ, 421,800
Mason, H. E., \& Monsignori Fossi, B. C. M. 1994, A\&AR, 6, 123

Mathioudakis, M., McKenny, J., Keenan, F. P., Williams, D. R., \& Phillips, K. J. H. 1999, A\&A, 351, L23

Mazzotta, P., Mazzitelli, G., Colafrancesco, S., \& Vittorio, N. 1998, A\&AS, 133, 403

McIntosh, S. W. 2000, ApJ, 533, 1043

Monsignori Fossi, B. C., \& Landini, M. 1991, Advances in Space Research, 11, 281

Monsignori Fossi, B. C., Landini, M., Del Zanna, G., \& Bowyer, S. 1996, ApJ, 466, 427

Pagano, I., Linsky, J. L., Carkner, L., et al. 2000, ApJ, 532, 497

Quin, D. A., Doyle, J. G., Butler, C. J., Byrne, P. B., \& Swank, J. H. 1993, A\&A, 272, 477

Raymond, J. C., Mazur, J. E., Allegrini, F., et al., 2001, in Solar and Galactic Composition, AIP Conf. Proc., 598, 49

Raymond, J. C., \& Doyle, J. G. 1981a, ApJ, 245, 1141

Raymond, J. C., \& Doyle, J. G. 1981b, ApJ, 247, 686

Raymond, J. C., \& Dupree, A. K. 1978, ApJ, 222, 379

Robinson, R. D., Linsky, J. L., Woodgate, B. E., \& Timothy, J. G. 2001, ApJ, 554, 368

Schmitt, J. H. M. M., Cutispoto, G., \& Krautter, J. 1998, ApJ, 500, L25

Tayal, S. S. 2000, ApJ, 530, 1091

Teriaca, L., Madjarska, M. S., \& Doyle, J. G. 2001, Sol. Phys., in press

Vernazza, J. E., \& Reeves, E. M. 1978, ApJS, 37, 485

Widing, K. G., \& Feldman, U. 1989, ApJ, 344, 1046

Young, P. R., Dupree, A. K., Wood, B. E., et al. 2001, ApJ, 555, L121

Zhang, H. L., Graziani, M., \& Pradhan, A. K. 1994, A\&A, 283, 319

Zhang, H. L., \& Sampson, D. H. 1992, Atomic Data and Nuclear Data Tables, 52, 143 